\title{
ARM EXPONENTS IN HIGH DIMENSIONAL PERCOLATION
}

\author{
GADY KOZMA AND ASAF NACHMIAS
}

\section{INTRODUCTION}

It is widely believed that there is no infinite component almost surely in critical percolation on any $d$-dimensional lattice for any $d>1$. Proving this is considered to be one of the most challenging problems in probability. This was proved for $d=2$ by Harris 21 and Kesten [26] and in high dimensions by Hara and Slade 19. By high dimensions we mean one of the two underlying graphs: (i) $\mathbb{Z}^{d}$ with $d \geq 19$ or, (ii) the graph with vertex set $\mathbb{Z}^{d}$ such that $x$ and $y$ are neighbors iff $|x-y| \leq L$ for sufficiently large $L$ and $d>6$ (see further definitions below).

Having no infinite component almost surely is equivalent to the assertion that the probability that the origin is connected by an open path to $\partial Q_{r}$, the boundary of the cube $\{-r, \ldots, r\}^{d}$ tends to 0 as $r \rightarrow \infty$. Physicists' lore (see for example [1], page 31) maintains that not only is there no infinite component for any $d \geq 2$, but also that these probabilities decay according to some power law in $r$; that is, $\mathbf{P}_{p_{c}}\left(0 \leftrightarrow \partial Q_{r}\right)=r^{-1 / \rho+o(1)}$ for some critical exponent $\rho>0$ which depends only on the dimension $d$, and not on the local structure of the lattice. In this paper we prove that $\rho=1 / 2$ in high dimensions.

Theorem 1. Consider critical percolation in high dimensions. Then we have

$$
\mathbf{P}_{p_{c}}\left(0 \leftrightarrow \partial Q_{r}\right) \approx r^{-2} .
$$

Here and below, $f(r) \approx g(r)$ means that for some constant $C>0$ which might depend on the dimension $d$ and on the specific lattice chosen, but not on $r$, we have $C^{-1} f(r) \leq g(r) \leq C f(r)$ for all $r>0$. A one-arm exponent was established in a few cases in the past.

- Kolmogorov 28 studied critical Galton-Watson processes and showed that for critical percolation on an infinite regular tree, $\rho=1$ (this can be considered as the $d=\infty$ case).

- In the breakthrough work of Lawler, Schramm and Werner 32, who relied on the work of Smirnov [39, 9], it is shown that $\rho=48 / 5$ for the triangular lattice in two dimensions.

- Van der Hofstad, den Hollander and Slade [24, 25] showed that $\rho=1$ in the setting of critical oriented spread-out percolation in dimension larger than 4 .

Even though most critical exponents for high dimensional percolation are known, the value of $\rho$ has remained undetermined. A previous attempt at calculating $\rho$ was made by Sakai [36]. He proved a conditional result implying that $\rho=1 / 2$, but

Received by the editors November 4, 2009 and, in revised form, July 21, 2010.

2010 Mathematics Subject Classification. Primary 60K35, 82B43.

(C)2010 American Mathematical Society Reverts to public domain 28 years from publication 
unfortunately his assumptions are not known to hold. One of his assumptions is that $\rho$ is well defined, an assertion we do not know how to prove without employing the full mechanism of this paper.

Rigorous results about critical percolation in high dimensions were obtained using the lace expansion, a perturbative technique inspired by the non-rigorous renormalization group methods used by physicists. We will liberally apply results achieved using the lace expansion, described below, but we do not use this technique directly.

1.1. Critical percolation in high dimensions. For an infinite graph $G$ and $p \in[0,1]$ we write $\mathbf{P}_{p}$ for the probability measure on subgraphs of $G$ obtained by independently retaining each edge with probability $p$ and deleting it with probability $1-p$. Edges retained are called open and edges deleted are called closed. The critical percolation probability $p_{c}$ is defined by

$$
\inf \left\{p: \mathbf{P}_{p}(\exists \text { an infinite component })>0\right\} .
$$

In this paper we consider critical percolation in high dimensions. By that we mean that $G=(V, E)$ is one of the following:

- the nearest neighbor model with $d \geq 19$, in which the vertex set $V=\mathbb{Z}^{d}$ and $E=\left\{(x, y):\|x-y\|_{1}=1\right\}$ or

- the spread-out model with $d>6$, in which $V=\mathbb{Z}^{d}$ and $E=\{(x, y)$ : $\left.\|x-y\|_{1} \leq L\right\}$ for some sufficiently large $L>L_{0}(d)$.

Informally, in high dimensions the space available for the critical percolation cluster to expand is so large that the interactions between different parts of the cluster become negligible, forming some independence between the different parts of the cluster. When the underlying graph is an infinite regular tree, this statement can be made completely formal. Indeed, the status of the edges descending from one branch of the root is independent of the status of the edges descending from another branch. Even though such strong independence does not hold in critical percolation on $\mathbb{Z}^{d}$, we still expect the same rough behavior when $d$ is large. One formal aspect of this heuristic is that we expect that the critical exponents, which describe the "shape" of the clusters, attain the same value as they do on an infinite regular tree.

A fundamental result in this spirit is due to Barsky and Aizenman [6] and Hara and Slade [19]. It states that in high dimensions we have

$$
\mathbf{P}_{p_{c}}(|\mathcal{C}(0)|>n) \approx \frac{1}{\sqrt{n}},
$$

where $\mathcal{C}(0)$ denotes the connected cluster containing the origin. It is a classical fact 5 that the same statement holds for critical percolation on an infinite regular tree. We remark that in [20] the precise asymptotic behavior of $\mathbf{P}_{p_{c}}(|\mathcal{C}(0)| \geq n)$ in high dimensions was obtained.

This appearance of "tree-like" behavior once the dimension is large occurs in many models of statistical physics. The dimension this transition occurs at is sometimes called the upper critical dimension. It is believed that for critical percolation, the upper critical dimension is 6. In particular, it is believed that (1.1) holds whenever $d>6$; however, this was proved only for the spread-out model and proving this in the full generality is still open.

In this paper we use the estimate (1.1) to prove our main theorem. Note, however, that we cannot expect Theorem 1 to hold assuming only (1.1) since in an 
infinite regular tree we have that (1.1) holds but $\rho=1$. At first, having $\rho=1 / 2$ in high dimensions may seem contradictory to the tree-like behavior mentioned above, but in fact, $\rho=1$ in a tree corresponds to $\rho^{\prime}=1$ in high dimensions, where $\rho^{\prime}$ is the intrinsic metric one-arm exponent. See [29] for more details.

The second estimate that we use, derived by Hara [17] (for the nearest-neighbor model) and by Hara, van der Hofstad and Slade [18. (for the spread-out model) states that in high dimensions,

$$
\mathbf{P}_{p_{c}}(0 \leftrightarrow x) \approx|x|^{2-d},
$$

where $0 \leftrightarrow x$ denotes the event that 0 is connected to $x$ with an open path. In fact, in [6] it is shown that this estimate implies (1.1). We may now state a more exact version of our result

Theorem 1 (conditional version). Assume $\left(\mathbb{Z}^{d}, E\right)$ is a lattice satisfying

(i) $d>6$;

(ii) the estimate (1.2);

(iii) the edge set $E$ is invariant under reflections and coordinate permutations.

Then

$$
\mathbf{P}_{p_{c}}\left(0 \leftrightarrow \partial Q_{r}\right) \approx r^{-2} .
$$

We explain why (iii) is needed in $\$ 1.3$

1.2. Outline of the proof. We use an induction scheme, not unlike the one used in [29, §3.2] for calculating the intrinsic one-arm exponent. Let us describe it in the roughest possible terms. Define $\gamma(r)=\mathbf{P}\left(0 \leftrightarrow \partial Q_{r}\right)$. Assume that the event $0 \leftrightarrow \partial Q_{3 r}$ occurred. Then one the the following must have happened.

(i) The cluster might have been not too small, that is, $|\mathcal{C}(0)| \geq \frac{1}{100} r^{4}$. By (1.1) this probability is at most $c / r^{2}$.

(ii) For every $j \in[r, 2 r]$, define

$$
X_{j}=\left|\left\{x \in \partial Q_{j}: 0 \stackrel{Q_{j}}{\longleftrightarrow} x\right\}\right|,
$$

where by $0 \stackrel{Q_{j}}{\longleftrightarrow} x$ we mean that 0 is connected to $x$ with an open path which resides in $Q_{j}$. The second possibility is that for some $j \in[r, 2 r]$ we have that $X_{j} \leq r^{2}$. For this to happen we must have that 0 is connected to $\partial Q_{j}$, which occurs with probability at most $\gamma(r)$, and then at least one $x \in \partial Q_{j}$ with $0 \stackrel{Q_{j}}{\longleftrightarrow} x$ must be connected to $\partial Q_{3 r}$, which costs us another $\gamma(r)$. Thus, the probability of this event is at most $r^{2} \gamma(r)^{2}$.

(iii) The remaining case is that $X_{j} \geq r^{2}$ for all $j \in[r, 2 r]$ and $\left|C_{0}\right| \leq \frac{1}{100} r^{4}$. Heuristically, if $X_{j} \geq r^{2}$ for all $j \in[r, 2 r]$, then we expect $|\mathcal{C}(0)|$ to be of size at least $r^{4}$. So the probability that $|\mathcal{C}(0)|$ is at most $\frac{1}{100} r^{4}$ should be small, say at most $\frac{1}{20}$. Remembering that we also need for 0 to be connected to $\partial Q_{r}$ we get that the probability of this possibility is at most $\frac{1}{20} \gamma(r)$.

All this gives the heuristic relation

$$
\gamma(3 r) \leq \frac{c}{r^{2}}+r^{2} \gamma(r)^{2}+\frac{1}{20} \gamma(r),
$$

from which it is possible to prove inductively that $\gamma(r)<C / r^{2}$. This is indeed the case, though we left out from this simplified sketch several additional parameters 
required for the induction to work. See the details in Chapter 2 below, starting with Lemma 2.3.

The estimate of (iii) is the hardest part. Let us therefore state it as a separate result. For this we need to introduce the following random variable. For $j \in[r, 2 r]$ and an integer $L \in[0, r]$ we define

$$
A_{j}=\left|\left\{y \in Q_{j+L} \backslash Q_{j}: 0 \leftrightarrow y\right\}\right| .
$$

Recall also the definition of $X_{j}$ at (1.3).

Theorem 2. There exists a constant $c>0$ such that for any $j$ sufficiently large, and any $L \geq j^{1 / 10}$ we have

$$
\mathbf{P}_{p_{c}}\left(X_{j} \geq L^{2} \text { and } A_{j} \leq c L^{4}\right) \leq(1-c) \mathbf{P}_{p_{c}}\left(0 \leftrightarrow \partial Q_{j}\right) .
$$

The exponent $1 / 10$ in the condition $L \geq j^{1 / 10}$ is immaterial and can be replaced with any positive number; however, this is unimportant since we apply this theorem with $L$ quite close to $j$.

Let us sketch the proof of Theorem 2 We condition on $X_{j}$ and then show using a second-moment method that $\mathbf{P}_{p_{c}}\left(A_{j}>c L^{4} \mid X_{j}\right)>c$. The main difficulty in the approach is the lower bound of the conditional first moment. Heuristically, each $x \in \partial Q_{j}$ "branches out" to $L^{2}$ vertices on average, so we should have $\mathbb{E}\left(A_{j} \mid X_{j}>\right.$ $\left.L^{2}\right) \geq L^{4}$, as long as

(i) the conditioning on $X_{j}$ does not alter significantly the behavior of one $x$, and

(ii) the different branches coming out of every $x$ do not intersect too much.

A natural approach to showing a claim of this sort would have been using the triangle condition. See [4, 6, 34, 36, 29, for details about the triangle condition and its applications. We could not make the triangle condition work directly, so we replaced it with a regularity analysis, which is similar in spirit, even if very different in detail. Let us expand on this topic.

1.3. Connection probability and cluster regularity. A key element in the proof is a lower bound on the connection probabilities. Let us state it formally.

Lemma 1.1. Let $\mathbb{Z}^{d}$ be a lattice in $\mathbb{R}^{d}$ such that the edge set $E\left(\mathbb{Z}^{d}\right)$ is symmetric with respect to coordinate permutations and reflections. Then there exist constants $C>0$ and $c>0$ such that for any $z \in \partial Q_{r}$ we have

$$
\mathbf{P}_{p_{c}}\left(0 \stackrel{Q_{r}}{\longleftrightarrow} z\right) \geq c e^{-C \log ^{2} r} .
$$

An interesting feature of this lemma is that it holds in all dimensions. However, the estimate is definitely not sharp, and we believe that the probability is in fact polynomially small and that it is minimized when $z$ sits in the corner of the cube, and then the probability is $\approx r^{\xi(d)}$ with $\xi(d)=2-2 d$ when $d>6$. The proof of this lemma is elementary, and it is there that we require the lattice to be invariant under coordinate permutations and reflections. Even though it is not sharp, Lemma 1.1 suffices to prove regularity results on the cluster of the origin. The precise form of regularity we need is somewhat technical and we expand on that in Chapter 4. Here let us demonstrate it with a simple example. Let $x \in \mathbb{Z}^{d}$ and define for any $A \subset \mathbb{Z}^{d}$,

$$
\mathcal{C}(x ; A)=\{y: x \stackrel{A}{\longleftrightarrow} y\} .
$$




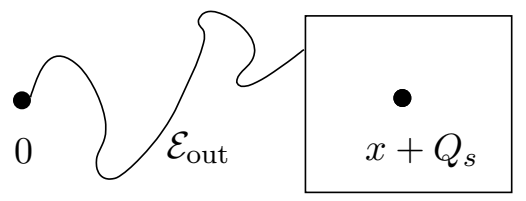

Figure 1 . The event $\mathcal{E}_{\text {out }}$ is independent of the edges in $x+Q_{s}$.

Let $s \geq 0$ and consider $\mathcal{C}\left(x ; x+Q_{s}\right)$, where $x+A$ is the translation $x+A=$ $\{x+y: y \in A\}$. It has been well known since 1984 4 that percolation clusters have an exponential tail beyond their typical "large" size, which is $s^{4}$ in our case. In other words,

$$
\mathbf{P}_{p_{c}}\left(\left|\mathcal{C}\left(x ; x+Q_{s}\right)\right| \geq s^{4} \log ^{3} s\right) \leq C e^{-c \log ^{3} s} .
$$

A regularity statement we wish to prove roughly asserts that the same bound (1.5) holds even if we know that $0 \leftrightarrow x$. Formally we wish to prove that

$$
\mathbf{P}_{p_{c}}\left(\left|\mathcal{C}\left(x ; x+Q_{s}\right)\right| \geq s^{4} \log ^{3} s \mid 0 \longleftrightarrow x\right) \leq C e^{-c \log ^{3} s} .
$$

What we need for the proof of Theorem 2 is somewhat different, but the idea is similar and Lemma 1.1 plays a crucial role.

To understand how the conditioning affects the picture let us now prove (1.6). Define $\mathcal{E}_{\text {out }}$ to be the event that 0 is connected to $x+Q_{s}$ (see Figure 11). We now apply Lemma 1.1. We get

$$
\mathbf{P}_{p_{c}}\left(0 \leftrightarrow x \mid \mathcal{E}_{\text {out }}\right) \geq c e^{-C \log ^{2} s},
$$

simply because conditioning on $\mathcal{E}_{\text {out }}$ reveals no information about the status of the edges in $x+Q_{s}$, and it is enough for $0 \leftrightarrow x$ to let $x$ connect to a single point on the boundary $x+\partial Q_{s}$. The former reason also shows that the events $\mathcal{E}_{\text {out }}$ and $\left|\mathcal{C}\left(x ; x+Q_{s}\right)\right| \geq s^{4} \log ^{3} s$ are independent. Hence,

$$
\begin{aligned}
\mathbf{P}_{p_{c}}(0 & \left.\longleftrightarrow x,\left|\mathcal{C}\left(x ; x+Q_{s}\right)\right| \geq s^{4} \log ^{3} s\right) \\
& \leq \mathbf{P}_{p_{c}}\left(\mathcal{E}_{\text {out }},\left|\mathcal{C}\left(x ; x+Q_{s}\right)\right| \geq s^{4} \log ^{3} s\right) \\
& =\mathbf{P}_{p_{c}}\left(\mathcal{E}_{\text {out }}\right) \mathbf{P}_{p_{c}}\left(\left|\mathcal{C}\left(x ; x+Q_{s}\right)\right| \geq s^{4} \log ^{3} s\right)
\end{aligned}
$$

By 1.5) $\leq \mathbf{P}_{p_{c}}\left(\mathcal{E}_{\text {out }}\right) C e^{-c \log ^{3} s}$

By (1.7) $\leq \mathbf{P}_{p_{c}}(0 \leftrightarrow x) C e^{-c \log ^{3} s+C \log ^{2} s} \leq C e^{-c \log ^{3} s} \mathbf{P}_{p_{c}}(0 \longleftrightarrow x)$,

which shows (1.6).

Inequality (1.6) is a local regularity assertion. From it one may get global regularity results in which similar estimates hold for most points of the cluster simultaneously. See the full details in Chapter 4 .

1.4. Multiple arms. The heuristic presented above suggests that the probability of having $\ell$ disjoint arms emanating from a small neighborhood of the origin is $r^{-2 \ell}$. This is indeed the content of the following theorem.

Theorem 3. For any integer $\ell \geq 1$ there exists a constant $K=K(\ell)$ such that for any $r>0$ and for any vertices $y_{1}, \ldots, y_{\ell} \in B\left(0, \frac{1}{2} r\right)$ satisfying $\left|y_{i}-y_{j}\right| \geq K$ for any $i \neq j$ we have

$$
\mathbf{P}_{p_{c}}\left(\left\{y_{1} \leftrightarrow \partial Q_{r}\right\} \circ \cdots \circ\left\{y_{\ell} \leftrightarrow \partial Q_{r}\right\}\right) \approx r^{-2 \ell},
$$


where the constants implied depend on $\ell, d$ and the specific lattice, but not on $r$.

The upper bound of this theorem follows immediately from the BK inequality; however, the lower bound requires an "inverse"-BK argument.

1.5. The BK-Reimer inequality. We close this introduction with a remark that might be interesting to some. In the proof of Lemma 5.5 we use Reimer's version of the van den Berg-Kesten inequality [35, 10. It does not seem as if the event at hand is an intersection of an increasing and a decreasing event, so one cannot replace it with the simpler van den Berg-Fiebig version 77. Nor did we see an obvious reduction to any simpler inequality. In short, it seems that the full power of Reimer's inequality is needed. In a similar spirit we drop the convention of using o for increasing events and $\square$ for general events, and use $\circ$ for both.

1.6. Notation. By "lattice" we mean a graph embedded in $\mathbb{R}^{d}$ such that the vertex set is $\mathbb{Z}^{d}$ and the edge set, which we shall denote by $E\left(\mathbb{Z}^{d}\right)$, is periodic with respect to a group of translations spanning $\mathbb{R}^{d}$ (by linear combinations). We assume the degree of each vertex is finite. We write $Q_{r} \subset \mathbb{Z}^{d}$ for the cube $\{-r, \ldots, r\}^{d}$ and $\partial Q_{r}$ for its internal boundary

$$
\partial Q_{r}=\left\{z \in Q_{r}: \exists x \notin Q_{r} \text { with }(z, x) \text { an edge in } \mathbb{Z}^{d}\right\} .
$$

We will not be very strict about $r$ being an integer, and in these cases we denote $Q_{r}=Q_{\lfloor r\rfloor}$, etc.

For two vertices $x, y$ we write $x \leftrightarrow y$ for the event that $x$ is connected to $y$ by an open path. It will be convenient to assume that $x \leftrightarrow x$ occurs always. We write $\mathcal{C}(x)$ for the connected component containing $x$, that is, $\mathcal{C}(x)=\{y: x \leftrightarrow y\}$. For a subset $A \subset \mathbb{Z}^{d}$ we write $x \stackrel{A}{\longleftrightarrow} y$ for the event that $x$ is connected to $y$ by an open path which is contained in $A$ (in particular, we must have $x, y \in A$ ) and we write $\mathcal{C}(x ; A)$ to denote the vertices connected to $x$ within $A$, that is, $\mathcal{C}(x ; A)=\{y: x \stackrel{A}{\longleftrightarrow} y\}$, as defined above. Notice that this usage of $x \stackrel{A}{\longleftrightarrow} y$ is different both from our use of it in our previous paper [29] and from that of Sakai [36]. We say that $x \leftrightarrow y$ off $A$ if there is an open path connecting $x$ and $y$ which avoids the vertices of $A$. We say that $x \leftrightarrow y$ only on $A$ if any open path between $x$ and $y$ intersects $A$. The last event is sometimes called $x \leftrightarrow y$ through $A$; see [19, 20].

For two events $\mathcal{A}, \mathcal{B}$ we write $\mathcal{A} \circ \mathcal{B}$ for the event that there exist two disjoint sets $U, V \subset E\left(\mathbb{Z}^{d}\right)$ such that the status of the edges of $U$ determines $\mathcal{A}$, and the status of the edges of $V$ determines $\mathcal{B}$. We frequently use the BK-Reimer inequality stating that $\mathbf{P}(\mathcal{A} \circ \mathcal{B}) \leq \mathbf{P}(\mathcal{A}) \mathbf{P}(\mathcal{B})$ (see [35, 10]) and the FKG inequality stating that if $\mathcal{A}$ and $\mathcal{B}$ are monotone increasing, then $\mathbf{P}(\mathcal{A} \cap \mathcal{B}) \geq \mathbf{P}(\mathcal{A}) \mathbf{P}(\mathcal{B})$.

For $x, y \in \mathbb{Z}^{d}$ we write $|x-y|$ for the Euclidean distance $\sqrt{\sum_{i}\left((x)_{i}-(y)_{i}\right)^{2}}$, $\|x-y\|_{\infty}$ for $\max \left|(x)_{i}-(y)_{i}\right|$ and $\|x-y\|_{1}$ for $\sum_{i}\left|(x)_{i}-(y)_{i}\right|$. For a subset of vertices $S \subset \mathbb{Z}^{d}$ and a vertex $x \in \mathbb{Z}^{d}$ we write $x+S$ for the translation $x+S=\{x+s: s \in S\}$. We denote by $c$ and $C$ positive constants which depend only on $d$ and on the specific lattice. The value of $c$ and $C$ will change from place to place, even within the same formula; occasionally we will number the constants $c_{1}, c_{2}, \ldots$ for clarity. Numbered constants do not change their value. We use $c$ for constants which are "small enough" and $C$ for constants which are "large enough". The notation $X \approx Y$ is short for $c X<Y<C X$. We did not make any attempt at optimizing constants in this work. Finally, let us remark on the use of $K$. We use $K$ consistently to denote 
a small translation or a small distance between two points. In a typical lemma, $K$ will start out as a free parameter, but will be fixed to a constant (depending on $d$ and the lattice) when enough information has been gathered. From that point on, we will consider it as just another $C$.

1.7. Organization. In the next chapter we show how to formalize the heuristic relation mentioned in $\$ 1.2$ and then, using Theorem 2 , perform the induction which yields the proof of Theorem 1 .

The majority of the paper is dedicated to proving Theorem 2. In Chapter 3 we prove Lemma 1.1. We use this in Chapter 4 to derive the regularity theorem, and apply all this to prove Theorem 2 in Chapter 5 . We conclude by proving Theorem 3 in Chapter 6 .

\section{The induction scheme: Proof of Theorem 1 using Theorem 2}

In this chapter we show how to derive our main result, Theorem 1 from Theorem 2. The difficulty in the proof of Theorem 1 is the upper bound. Indeed, the lower bound on $\mathbf{P}\left(0 \leftrightarrow \partial Q_{r}\right)$ follows from a simple second moment estimate using the 2-point function estimate (1.2). This will be proved in Lemma 2.2 right after the following simple calculation.

Lemma 2.1. There exists a constant $C>0$ such that for any $r$ we have

$$
\sum_{x, y \in Q_{r}} \mathbf{P}(0 \leftrightarrow x, 0 \leftrightarrow y) \leq C r^{6}
$$

Proof. If $x$ and $y$ are connected to 0 , then there exists $z$ such that the events $\{0 \leftrightarrow z\},\{z \leftrightarrow x\}$ and $\{z \leftrightarrow y\}$ occur disjointly (we allow the case $z=0$ ). This is easy to see, and [16, proof of theorem (6.75)] gives a careful derivation. By the BK inequality and the two-point function estimate (1.2) we get that

$$
\sum_{x, y \in Q_{r}} \mathbf{P}(0 \leftrightarrow x, 0 \leftrightarrow y) \leq \sum_{x, y \in Q_{r}, z \in \mathbb{Z}^{d}} \mathbf{P}(\{0 \leftrightarrow z\} \circ\{z \leftrightarrow x\} \circ\{z \leftrightarrow y\})
$$

by BK

$$
\leq \sum_{x, y \in Q_{r}, z \in \mathbb{Z}^{d}} \mathbf{P}(0 \leftrightarrow z) \mathbf{P}(z \leftrightarrow x) \mathbf{P}(z \leftrightarrow y)
$$

by (1.2)

$$
<C \sum_{x, y \in Q_{r}, z \in \mathbb{Z}^{d}}|z|^{2-d}|x-z|^{2-d}|y-z|^{2-d} .
$$

We estimate this sum in two parts. For $|z| \leq d r$ we simply sum over $y$, then over $x$ and finally over $z$ to get

$$
\sum_{x, y \in Q_{r},|z| \leq d r}|z|^{2-d}|x-z|^{2-d}|y-z|^{2-d}<C r^{6} .
$$

In the other case, $|z|>d r$, we have $|z|>2|x|$ because $|x| \leq r \sqrt{d}$ and $d>6$ so $|z-x|>|z| / 2$ and $|z-y|>|z| / 2$. Hence

$$
\sum_{x, y \in Q_{r},|z| \geq d r}|z|^{2-d}|x-z|^{2-d}|y-z|^{2-d}<C r^{2 d} \sum_{|z|>d r}|z|^{6-3 d}<C r^{6} .
$$

Lemma 2.2. There exists some constant $c>0$ such that

$$
\mathbf{P}\left(0 \leftrightarrow \partial Q_{r}\right) \geq \frac{c}{r^{2}},
$$

for all $r>0$. 
Proof. Define the random variable $X$ by

$$
X=\left|\left\{x \in Q_{2 r} \backslash Q_{r}: 0 \leftrightarrow x\right\}\right| .
$$

By the 2-point function estimate (1.2) we have

$$
\mathbb{E} X>c r^{d} r^{2-d}=c r^{2} .
$$

The second moment is bounded by Lemma2.1 so $\mathbb{E} X^{2} \leq C r^{6}$. Observe that $X>0$ implies that $0 \leftrightarrow \partial Q_{r}$ and hence we get

$$
\mathbf{P}\left(0 \leftrightarrow \partial Q_{r}\right) \geq \frac{(\mathbb{E} X)^{2}}{\mathbb{E} X^{2}} \geq c r^{-2} .
$$

We move to our main endeavor, that of proving Theorem 1 from Theorem 2 First we get from Theorem 2 a recursive inequality for $\mathbf{P}\left(0 \leftrightarrow \partial Q_{r}\right)$. Let us state it as a lemma.

Lemma 2.3. Write $\gamma(r)=\mathbf{P}\left(0 \leftrightarrow \partial Q_{r}\right)$. There exist positive constants $c_{1}$ and $C_{1}$ such that for all $\lambda \in(0,1]$ there exists $\epsilon_{0}=\epsilon_{0}(\lambda)$ such that for all $\epsilon \in\left(0, \epsilon_{0}\right)$ we have

$$
\gamma(r(1+\lambda)) \leq \frac{C_{1}}{\sqrt{\epsilon} r^{2}}+\epsilon^{3 / 5} r^{2} \gamma(r) \gamma\left(\frac{\lambda r}{2}\right)+\left(1-c_{1}\right) \gamma(r) .
$$

Remark. The value $\frac{3}{5}$ is somewhat arbitrary, but the proof of Theorem 1 requires that it would be larger than $\frac{1}{2}$.

Proof. Let us first dispose of an uninteresting range of parameters, the case that $\epsilon \leq 2 r^{-3}$. In this case we simply use Barsky-Aizenman (1.1) and get

$$
\gamma(r(1+\lambda)) \stackrel{1.1}{\leq} \frac{C}{\sqrt{r}} \leq \frac{C}{\sqrt{\epsilon} r^{2}}
$$

and we are done (with no need to examine the other terms in (2.1)).

Otherwise, define $L=\epsilon^{3 / 10} r$. Recall the definitions of $X_{j}$ and $A_{j}$ in (1.3), (1.4) preceding the statement of Theorem 2 (with the $L$ just defined). If $0 \leftrightarrow \partial Q_{r(1+\lambda)}$, then one of the following events must occur:

(i) $|\mathcal{C}(0)| \geq \epsilon r^{4}$.

(ii) For some $j \in[r(1+\lambda / 4), r(1+\lambda / 2)]$ we have $X_{j} \leq L^{2}$ and $0 \leftrightarrow \partial Q_{r(1+\lambda)}$.

(iii) For all $j \in[r(1+\lambda / 4), r(1+\lambda / 2)]$ we have $X_{j}>L^{2}$ and $|\mathcal{C}(0)|<\epsilon r^{4}$.

Denote these events by $\mathcal{B}_{1}, \mathcal{B}_{2}$ and $\mathcal{B}_{3}$ respectively.

The term $\mathcal{B}_{1}$. By Barsky-Aizenman (1.1) we bound

$$
\mathbf{P}\left(\mathcal{B}_{1}\right) \leq \frac{C_{1}}{r^{2} \sqrt{\epsilon}},
$$

which gives the first term in (2.1).

The term $\mathcal{B}_{2}$. We estimate $\mathbf{P}\left(\mathcal{B}_{2}\right)$ using a regeneration argument similar to the one used in [29, eq. (3.8)]. Let $j_{0} \in[r(1+\lambda / 4), r(1+\lambda / 2)]$ be the first $j$ for which $0<X_{j} \leq L^{2}$, and condition on $\mathcal{C}=\mathcal{C}\left(0 ; Q_{j_{0}}\right)$. We get

$$
\mathbf{P}\left(\mathcal{B}_{2}\right)=\sum_{A \text { admissable }} \mathbf{P}(\mathcal{C}=A) \mathbf{P}\left(0 \leftrightarrow \partial Q_{r(1+\lambda)} \mid \mathcal{C}=A\right),
$$


where "admissible" means that $\mathbf{P}(\mathcal{C}=A)>0$. If $0 \leftrightarrow \partial Q_{r(1+\lambda)}$, then one of the vertices of $\partial \mathcal{C}$ must be connected to $\partial Q_{r(1+\lambda)}$ off $\mathcal{C}$, so we can write

$$
\mathbf{P}\left(0 \leftrightarrow \partial Q_{r(1+\lambda)} \mid \mathcal{C}=A\right) \leq \sum_{x \in A \cap \partial Q_{j_{0}}} \mathbf{P}\left(x \leftrightarrow \partial Q_{r(1+\lambda)} \text { off } A \mid \mathcal{C}=A\right) .
$$

We now note that $\mathcal{C}\left(0 ; Q_{j}\right)$ allows us to tell whether $j=j_{0}$ or not; no information from the rest of the configuration is needed (here it is important that $j_{0}$ is the first such $j)$. Therefore the conditioning over $\mathcal{C}\left(0 ; Q_{j_{0}}\right)=A$ gives no information on the rest of the configuration and we learn that

$$
\begin{aligned}
\mathbf{P}\left(x \leftrightarrow \partial Q_{r(1+\lambda)} \text { off } A \mid \mathcal{C}=A\right) & =\mathbf{P}\left(x \leftrightarrow \partial Q_{r(1+\lambda)} \text { off } A\right) \\
& \leq \mathbf{P}\left(x \leftrightarrow \partial Q_{r(1+\lambda)}\right) \\
\text { since } x \in \partial Q_{j_{0}} \subset Q_{r(1+\lambda / 2)} & \leq \gamma\left(\frac{\lambda r}{2}\right) .
\end{aligned}
$$

The sum over all $x$ gives a factor of at most $L^{2}$ by definition of $j_{0}$. Plugging this into (2.2) gives

$$
\mathbf{P}\left(\mathcal{B}_{2}\right) \leq L^{2} \gamma\left(\frac{\lambda r}{2}\right) \sum_{A \text { admissable }} \mathbf{P}(\mathcal{C}=A) \leq L^{2} \gamma\left(\frac{\lambda r}{2}\right) \gamma(r),
$$

which is the second term in (2.1).

The term $\mathcal{B}_{3}$. It is at this point that we use Theorem 2 Let us first verify the conditions of the theorem, namely that $j$ is sufficiently large and that $L \geq j^{1 / 10}$. We may definitely assume that $r$ is sufficiently large because for small $r$ setting $C_{1}$ large will render the lemma true vacuously. Also, $j>r$. For the second condition we recall that at the very beginning of the lemma we assumed $\epsilon>2 r^{-3}$ and then $L>(8 r)^{1 / 10}>j^{1 / 10}$ (here is where we used $\lambda \leq 1$ to make sure $j \leq 2 r$ ). Hence we may apply Theorem 2 For every integer $1 \leq i \leq \frac{1}{4} \lambda \epsilon^{-3 / 10}$ let

$$
j_{i}=r+\frac{1}{4} \lambda r+i L \in\left[r\left(1+\frac{1}{4} \lambda\right), r\left(1+\frac{1}{2} \lambda\right)\right] .
$$

Let $c_{2}$ be the constant from Theorem 2. We define the random variable

$$
I=\mid\left\{i: X_{j_{i}} \geq L^{2} \text { and } A_{j_{i}}<c_{2} L^{4}\right\} \mid .
$$

Now, if $|\mathcal{C}(0)|<\epsilon r^{4}$, then

$$
\left|\left\{i: A_{j_{i}} \geq c_{2} L^{4}\right\}\right|<\frac{\epsilon r^{4}}{c_{2} L^{4}}=\frac{\epsilon^{-1 / 5}}{c_{2}} .
$$

However, $\mathcal{B}_{3}$ implies that all $X_{j_{i}} \geq L^{2}$ and hence

$$
\mathcal{B}_{3} \Rightarrow I>\left\lfloor\frac{1}{4} \lambda \epsilon^{-3 / 10}\right\rfloor-c_{2}^{-1} \epsilon^{-1 / 5} .
$$

This last formula is the most interesting restriction on the exponent $3 / 5$ in the statement of the lemma. We need it here to be less than 2/3; otherwise the term subtracted would be bigger than the positive term, rendering the estimate useless.

On the other hand, summing the estimate of Theorem 2 over all $i$ gives that

$$
\mathbb{E} I \leq\left(1-c_{2}\right) \gamma(r) \frac{1}{4} \lambda \epsilon^{-3 / 10}
$$


and hence by Markov's inequality,

$$
\begin{aligned}
\mathbf{P}\left(\mathcal{B}_{3}\right) \leq \mathbf{P}\left(I>\left\lfloor\frac{1}{4} \lambda \epsilon^{-3 / 10}\right\rfloor-c_{2}^{-1} \epsilon^{-1 / 5}\right) & \leq \frac{\left(1-c_{2}\right) \frac{1}{4} \lambda \epsilon^{-3 / 10}}{\frac{1}{4} \lambda \epsilon^{-3 / 10}-c_{2}^{-1} \epsilon^{-1 / 5}-1} \gamma(r) \\
& \leq \frac{1-c_{2}}{1-C \lambda^{-1} \epsilon^{1 / 10}} \gamma(r),
\end{aligned}
$$

and with $\epsilon$ sufficiently small, depending on $\lambda$, this is at most $\left(1-c_{1}\right) \gamma(r)$, say, with $c_{1}:=\frac{1}{2} c_{2}$. This is the last term in (2.1), and the lemma is proved.

Proof of Theorem 11. Let $c_{1}$ and $C_{1}$ be as in Lemma 2.3. We first fix $\lambda>0$ sufficiently small that

$$
(1+\lambda)^{2} \leq 2, \quad\left(1-c_{1}\right)(1+\lambda)^{2} \leq\left(1-\frac{1}{2} c_{1}\right) .
$$

Next we fix $M$ so large that

$$
\begin{aligned}
\left(2 C_{1}+8 \lambda^{-2}\right) M^{-1 / 11} & \leq \frac{1}{2} c_{1}, \\
M^{-20 / 11} & \leq \epsilon_{0}(\lambda),
\end{aligned}
$$

where $\epsilon_{0}(\lambda)$ is also from the statement of Lemma 2.3 . We shall prove by induction that for any $r$ we have $\gamma(r) \leq M r^{-2}$. For convenience of notation, assume we wish to prove the claim for $r(1+\lambda)$, so the induction assumption is

$$
\gamma(s) \leq \frac{M}{s^{2}} \quad \forall s<r(1+\lambda) .
$$

We now use Lemma 2.3 with $\epsilon=M^{-20 / 11}$ (here is where we need (2.5)) and get

$$
\begin{aligned}
\gamma(r(1+\lambda)) & \leq \frac{C_{1}}{\sqrt{\epsilon} r^{2}}+\epsilon^{3 / 5} r^{2} \gamma(r) \gamma\left(\frac{\lambda r}{2}\right)+\left(1-c_{1}\right) \gamma(r) \\
\text { inductively } & \leq \frac{C_{1} M^{10 / 11}}{r^{2}}+M^{-12 / 11} r^{2} \cdot \frac{M}{r^{2}} \cdot \frac{4 M}{(\lambda r)^{2}}+\left(1-c_{1}\right) \frac{M}{r^{2}} \\
\text { by (2.3) } & \leq \frac{M}{(r(1+\lambda))^{2}}\left(M^{-1 / 11}\left(2 C_{1}+8 \lambda^{-2}\right)+\left(1-\frac{1}{2} c_{1}\right)\right) \\
\text { by (2.4) } & \leq \frac{M}{(r(1+\lambda))^{2}} .
\end{aligned}
$$

This concludes the proof of the theorem.

\section{A lower bound on Connection probability: Proof of Lemma 1.1}

In this chapter we assume neither that $d>6$ nor that (1.1) or (1.2) hold.

Lemma 3.1. Let $\mathbb{Z}^{d}$ be a bounded lattice in $\mathbb{R}^{d}$. Then, for any $p \geq p_{c}$ and any $r>0$

$$
\sum_{z \in \partial Q_{r}} \mathbf{P}\left(0 \stackrel{Q_{r}}{\longrightarrow} z\right) \geq 1
$$

Proof. Assume the contrary and let $\epsilon>0$ and $r>0$ be such that $\sum_{z \in \partial Q_{r}} \mathbf{P}\left(0 \stackrel{Q_{r}}{\longleftrightarrow}\right.$ $z)=1-\epsilon$. We will show that

$$
\mathbb{E}|\mathcal{C}(0)|<\infty .
$$

It is well known that this implies that $p<p_{c}$; see [4, eq. (3.2)] or [16]. Hence the lemma will be proved once we demonstrate (3.1). 

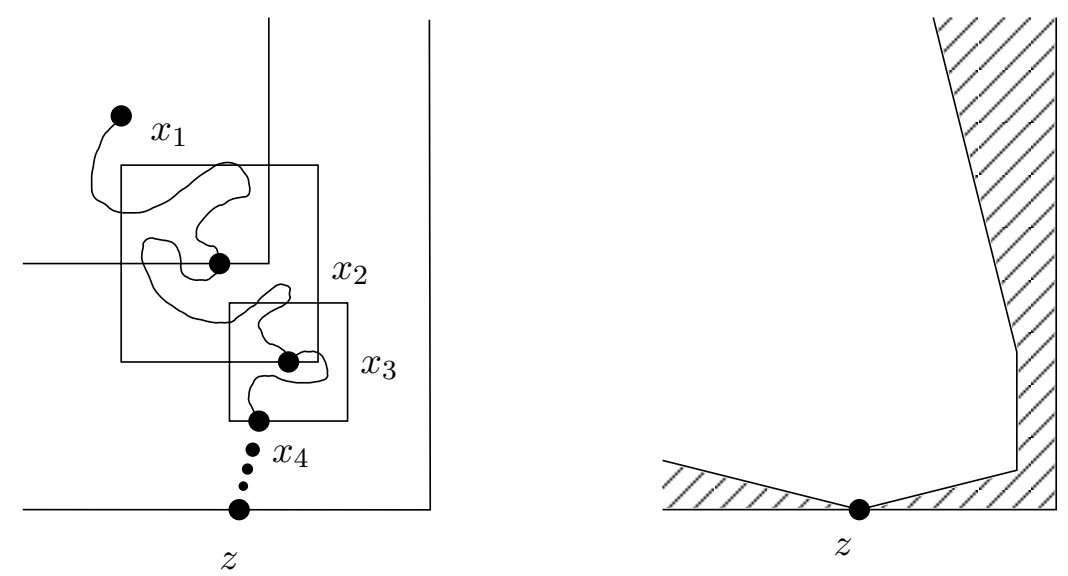

Figure 2. On the left, the points $x_{i}$. On the right, the forbidden area for each $x_{i}$.

To see (3.1) fix an integer $n$. Let $\mathcal{X}_{n}$ be the collection of $n$-tuples $0=x_{1}, \ldots, x_{n}$ satisfying that:

(i) $x_{i+1} \in x_{i}+\partial Q_{r}$.

(ii) There exist open simple paths $\gamma_{i}$ from $x_{i}$ to $x_{i+1}$ with $\gamma_{i} \subset x_{i}+Q_{r}$.

(iii) The $\gamma_{i}$ are vertex-disjoint except at their end-points.

By the BK inequality and translation invariance we have

$$
\mathbf{P}\left(\left(x_{1}, \ldots, x_{n}\right) \in \mathcal{X}_{n}\right) \leq \prod_{i=1}^{n} \mathbf{P}\left(0 \leftrightarrow x_{i}-x_{i-1} \text { in } Q_{r}\right)
$$

and summing over all possible $n$-tuples $\left(x_{1}, \ldots, x_{n}\right)$ gives

$$
\mathbf{P}\left(\mathcal{X}_{n} \neq \emptyset\right) \leq\left(\sum_{z \in \partial Q_{r}} \mathbf{P}\left(0 \leftrightarrow z \text { in } Q_{r}\right)\right)^{n}=(1-\epsilon)^{n}
$$

by our contradictory assumption.

Now fix some $z \notin Q_{n r}$. If $0 \leftrightarrow z$, then there must exist some open simple path $\gamma: 0 \rightarrow z$. Define $x_{1}=0$ and then inductively $x_{i+1}$ to be the first point on $\gamma$ after $x_{i}$ in $x_{i}+\partial Q_{r}$. Clearly this process lasts at least $n$ steps. Hence $0 \leftrightarrow z$ implies $\mathcal{X}_{n} \neq \emptyset$ and, in particular,

$$
\mathbf{P}(0 \leftrightarrow z) \leq(1-\epsilon)^{n} .
$$

So

$$
\mathbb{E}\left(\left|\mathcal{C}(0) \cap\left(Q_{(n+1) r} \backslash Q_{n r}\right)\right|\right) \leq(2(n+1) r)^{d}(1-\epsilon)^{n}
$$

and summing over $n$ gives (3.1) and finishes the lemma.

Proof of Lemma 1.1. We shall construct a sequence of cubes $x_{i}+Q_{M_{i}} \subset Q_{r}$, for $i=1, \ldots, N$ and $N \leq C \log r$, such that $x_{1}=0$ but $x_{N}=z$ and

$$
\mathbf{P}\left(x_{i} \stackrel{x_{i}+Q_{M_{i}}}{\longleftrightarrow} x_{i+1}\right) \geq c r^{1-d} .
$$


See Figure 2 left. This will of course finish the lemma, by the FKG inequality:

$$
\begin{aligned}
\mathbf{P}\left(0 \stackrel{Q_{r}}{\longleftrightarrow} z\right) & \geq \mathbf{P}\left(x_{1} \stackrel{Q_{r}}{\longleftrightarrow} x_{2}, \ldots, x_{N-1} \stackrel{Q_{r}}{\longleftrightarrow} x_{N}\right) \\
& \geq \prod_{i=1}^{N-1} \mathbf{P}\left(x_{i} \stackrel{Q_{r}}{\longleftrightarrow} x_{i+1}\right) \\
\text { by FKG } & \geq \prod_{i=1}^{N-1} \mathbf{P}\left(x_{i} \stackrel{x_{i}+Q_{M_{i}}}{\longleftrightarrow} x_{i+1}\right) \\
\text { because } x_{i}+Q_{M_{i}} \subset Q_{r} & \geq \prod_{i=1}^{N-1} c r^{1-d} \geq c e^{-C \log ^{2} r} .
\end{aligned}
$$

Hence we only need to construct the $x_{i}$.

The construction is inductive, and it is important to keep the $x_{i}$ away from the boundary of $Q_{r}$ throughout the process; otherwise we would not be able to choose a reasonably big $M_{i}$ with $x_{i}+Q_{M_{i}} \subset Q_{r}$. Hence we will require that for every index $1 \leq j \leq d$,

$$
r-\left|\left(x_{i}\right)_{j}\right| \geq \frac{1}{4}\left\|z-x_{i}\right\|_{\infty}
$$

where $\left(x_{i}\right)_{j}$ is the $j$-th coordinate of $x_{i}$. See Figure 2 right.

We proceed to the details of the construction. Assume $x_{1}, \ldots, x_{i}$ have already been defined. Define $M_{i}=\frac{1}{4}\left\|z-x_{i}\right\|_{\infty}$. By assumption (3.3), $x_{i}+Q_{M_{i}} \subset Q_{r}$, as required. By Lemma 3.1 we have

$$
\sum_{y \in \partial Q_{M_{i}}} \mathbf{P}\left(0 \stackrel{Q_{M_{i}}}{\longleftrightarrow} y\right) \geq 1
$$

and therefore there exists some $y$ such that

$$
\mathbf{P}\left(0 \stackrel{Q_{M_{i}}}{\longleftrightarrow} y\right) \geq c M_{i}^{1-d} \geq c r^{1-d} .
$$

We want to define $x_{i+1}=x_{i}+y$ but that might take us in the wrong direction, that is, not towards $z$. It is at this point that we use the symmetries of the lattice. The symmetries allow us to rearrange the coordinates of $y$ and change their signs and (3.4) will still hold. We do so according to the following rules:

(i) $\left|y_{j}\right|$ are arranged like $\left|\left(z-x_{i}\right)_{j}\right|$; i.e., if $\left|\left(z-x_{i}\right)_{j}\right|>\left|\left(z-x_{i}\right)_{k}\right|$, then $\left|y_{j}\right| \geq\left|y_{k}\right|$.

(ii) In directions $j$ where $\left|\left(z-x_{i}\right)_{j}\right| \geq 2 M_{i}$ we want $x_{i+1}$ to be closer to $z$, so set $\operatorname{sign} y_{j}=\operatorname{sign}\left(z-x_{i}\right)_{j}$. We will see later that this automatically takes care of the distance from $\partial Q_{r}$.

(iii) Otherwise we ignore the distance from $z$ and just pull away from $\partial Q_{r}$; i.e., set $\operatorname{sign} y_{j}=-\operatorname{sign}\left(x_{i}\right)_{j}$. For notational convenience, assume here and below that $\operatorname{sign} 0=1$.

This concludes the description of the construction, and we automatically get the connection probability estimate (3.2).

Next we wish to verify that we indeed reach a neighbor of $z$ in at most $C \log r$ steps and that (3.3) holds. We shall show that every step of the induction does not 
increase $\left\|z-x_{i}\right\|_{\infty}$ and after $d$ steps the norm is reduced by a constant, i.e.,

$$
\left\|z-x_{i+d}\right\|_{\infty} \leq \frac{3}{4}\left\|z-x_{i}\right\|_{\infty}
$$

which is enough. We first note that by (ii) the fact that $\|y\|_{\infty} \leq M_{i}$ and that $\left\|z-x_{i}\right\|_{\infty}=4 M_{i}$, it is immediately clear that

$$
\left\|z-x_{i+1}\right\|_{\infty} \leq\left\|z-x_{i}\right\|_{\infty}
$$

Further, since $y \in \partial Q_{M_{i}}$, then it must have at least one coordinate with absolute value $M_{i}$. Denote by $j_{1}$ the largest coordinate in absolute value of $z-x_{i}$. We get that $\left|\left(z-x_{i}\right)_{j_{1}}\right|$ is reduced from $4 M_{i}$ to $3 M_{i}$. Again from (ii) we see that at the next steps it will stay below $3 M_{i}$, because it can only increase (at some step $i+k$ ) if it becomes $\leq 2 M_{i+k}$ and in this case it can only increase up to $3 M_{i+k}$. This is $\leq 3 M_{i}$ by (3.6). In short we get

$$
\left|\left(z-x_{i+k}\right)_{j_{1}}\right| \leq 3 M_{i} \quad \forall k \geq 1 .
$$

Next denote by $j_{2}$ the largest coordinate of $z-x_{i+1}$. If $j_{2}=j_{1}$, then $\left\|z-x_{i+1}\right\|_{\infty} \leq$ $3 M_{i}$ and (3.5) is proved. Otherwise we get from the same arguments,

$$
\left|\left(z-x_{i+1+k}\right)_{j_{2}}\right| \leq 3 M_{i+1} \leq 3 M_{i} \quad \forall k \geq 1,
$$

and so on. By step $i+d$ we would have either covered all coordinates or run into a case of two equal $j$ 's, either of which demonstrates (3.5) and hence that $N \leq C \log r$.

To complete the induction we need to show that (3.3) is preserved. Clearly it holds for $i=0$. For $i>1$ we have two cases:

The case $\left|\left(z-x_{i}\right)_{j}\right|<2 M_{i}$. In this case, by (iii), we try to increase the distance from $r$, and we succeed unless $\left|\left(x_{i}\right)_{j}\right|<\frac{1}{2}\left|\left(y_{i}\right)_{j}\right|$. If we succeed, then

$$
r-\left|\left(x_{i+1}\right)_{j}\right| \geq r-\left|\left(x_{i}\right)_{j}\right| \stackrel{3.31}{\geq} M_{i} \stackrel{3.6}{\geq} M_{i+1},
$$

where the reference to 3.3 in the formula above is a reference to our inductive assumption of the validity of (3.3) in the previous step. If we failed, then

$$
r-\left|\left(x_{i+1}\right)_{j}\right| \geq r-\left|\left(y_{i}\right)_{j}\right| \geq 4 M_{i}-M_{i} \stackrel{\sqrt[3.6]{>}}{>} M_{i+1} .
$$

The case $\left|\left(z-x_{i}\right)_{j}\right| \geq 2 M_{i}$. If adding $y$ increases the distance of $x$ to $\partial Q_{r}$, then the argument of (3.8) applies with no change. If not, then we must have that

$$
\left(x_{i}\right)_{j} \cdot \operatorname{sign}\left((z)_{j}\right) \in\left[-\frac{1}{2}\left|y_{j}\right|,\left|(z)_{j}\right|-2 M_{i}\right],
$$

SO

$$
\left(x_{i+1}\right)_{j} \cdot \operatorname{sign}\left((z)_{j}\right) \in\left[\frac{1}{2}\left|y_{j}\right|,\left|(z)_{j}\right|-M_{i}\right]
$$

But in this case,

$$
r-\left|\left(x_{i+1}\right)_{j}\right| \geq M_{i} \stackrel{3.6}{\geq} M_{i+1} .
$$

Together with (3.8) this shows that (3.3) is preserved inductively and hence holds for all $i$. This shows that the induction is valid and proves the lemma. 


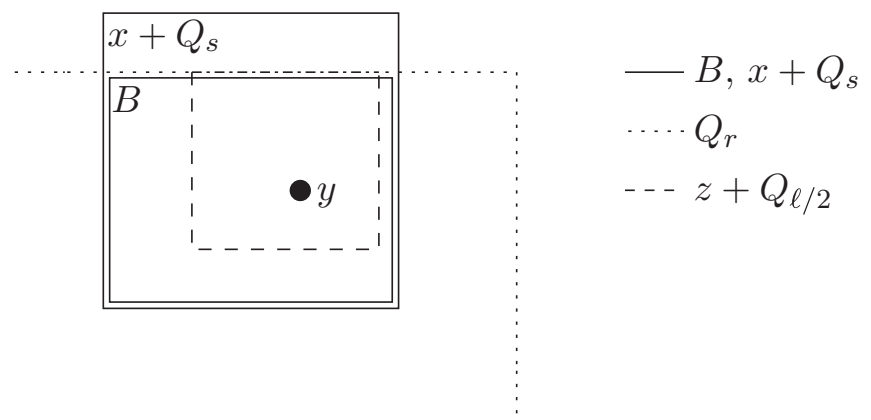

Figure 3. Corollary 3.2

Corollary 3.2. Let $r>s>0$ and let $x \in \mathbb{Z}^{d}$ such that

$$
\left(x+Q_{s}\right) \cap \partial Q_{r} \neq \emptyset .
$$

Then for any $y \in B:=\left(x+Q_{s}\right) \cap Q_{r}$,

$$
\mathbf{P}\left(y \stackrel{B}{\leftrightarrow} \partial Q_{r}\right) \geq e^{-c \log ^{2} s} .
$$

See Figure 3

Proof. $B$ is a box (i.e., with the sides parallel to the axis, but their lengths not necessarily equal). Denote by $\ell$ its shortest edge so that $\ell \leq 2 \mathrm{~s}$. It is now easy to see that one can find a cube $z+Q_{\ell / 2} \subset B$ containing both $y$ and at least one point from $\partial Q_{r}$; just construct $z$ coordinate by coordinate and they are independent. Now write

by FKG

$$
\mathbf{P}\left(y \stackrel{B}{\longleftrightarrow} \partial Q_{r}\right) \geq \mathbf{P}\left(y \stackrel{B}{\longleftrightarrow} z \text { and } z \stackrel{B}{\longleftrightarrow} \partial Q_{r}\right)
$$$$
\geq \mathbf{P}(y \stackrel{B}{\longleftrightarrow} z) \mathbf{P}\left(z \stackrel{B}{\longleftrightarrow} \partial Q_{r}\right)
$$

by Lemma 1.1

$$
\geq \mathbf{P}\left(y \stackrel{z+Q_{\|y-z\| \infty}}{\longleftrightarrow} z\right) \mathbf{P}\left(z \stackrel{z+Q_{\ell / 2}}{\longleftrightarrow} \partial Q_{r}\right)
$$

$$
\geq c \exp \left(-C \log ^{2}\|y-z\|_{\infty}\right) \exp \left(-C \log ^{2}(\ell / 2)\right)
$$$$
\geq c \exp \left(-C \log ^{2} s\right) \text {, }
$$

as required.

\section{A regularity theorem}

In the following we prove a regularity result which is the key element in the proof of Theorem 2 in Chapter 5 . We recommend that the reader first read 4.1 . containing the required definitions and the statement of the theorem, then read how it is used in Chapter 5 and especially in Lemma 5.5 before returning to the proof of the regularity theorem, which is the bulk of this chapter.

4.1. Statement of the regularity theorem. We are interested in estimating the tails of random variables of the form $\left|\mathcal{C}(x) \cap Q_{s}\right|$. For any particular $x$ this can easily be done using (1.2), the BK inequality and a moment calculation. In fact, this is exactly performed in [2. Let us therefore define the event that the cluster is "typical",

$$
\mathcal{T}_{s}(x)=\left\{\left|\mathcal{C}(x) \cap\left(x+Q_{s}\right)\right|<s^{4} \log ^{7} s\right\} .
$$



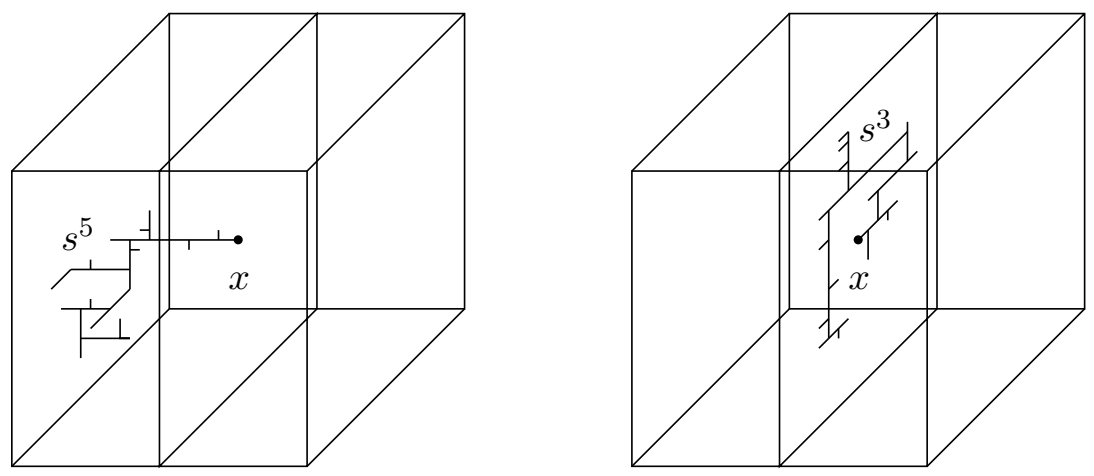

Figure 4. Two kinds of bad configuration. On the left, a simple bad configuration with too many vertices inside the left half-cube. On the right, a bad configuration with too many vertices on the surface of the half-cube.

As discussed in the introduction (see (1.5)), $\mathbf{P}\left(\mathcal{T}_{s}(x)\right)>1-e^{-c \log ^{7} s}$. Where we deviate from the simplified sketch in the introduction is in the following definition:

Definition 4.1. For $x \in \partial Q_{j}$ and positive integers $s$ and $K$ we define the following events.

(i) We say that $x$ is $s$-bad if $\mathcal{C}\left(x ; Q_{j}\right)$ satisfies

$$
\mathbf{P}\left(\mathcal{T}_{s}(x) \mid \mathcal{C}\left(x ; Q_{j}\right)\right) \leq 1-\exp \left(-\log ^{2} s\right) .
$$

(ii) We say that $x \in \partial Q_{j}$ is $K$-irregular if there exists $s \geq K$ such that $x$ is $s$-bad. Otherwise we say that $x$ is $K$-regular.

The notation "| $\mathcal{C}\left(x ; Q_{j}\right)$ " means that we condition on all open edges between two vertices of the cluster $\mathcal{C}\left(x ; Q_{j}\right)$ as well as on all closed edges with both vertices in $Q_{j}$ and at least one vertex in $\mathcal{C}\left(x ; Q_{j}\right)$. In short, we condition on all information needed to calculate $\mathcal{C}\left(x ; Q_{j}\right)$ precisely. Note that we do not condition on edges leading outside of $Q_{j}$.

Let us briefly discuss the significance of Definition 4.1. Typically $j$ is large and $s$ is $j^{o(1)}$. Clearly the event that $x$ is $s$-bad is unusual, due to the power of the log being 2 in (4.2) and 7 in (4.1). The event that $x$ is $s$-bad depends on the status of edges in $\mathcal{C}\left(x ; Q_{j}\right)$ and indirectly reveals that the boundary of the cluster (at $\partial Q_{j}$ ) is sufficiently spread out. This is best illustrated by the following two examples of bad configurations.

The first is a "simple" bad configuration. See Figure 4 , left. In this case the configuration $\mathcal{C}\left(x ; Q_{j}\right)$ has a cluster of size at least $s^{4} \log ^{7} s$ inside $\left(x+Q_{s}\right) \cap Q_{j}$, so the conditional probability in (4.2) is just 0 . The second, and more interesting (see Figure 4, right) is when the configuration has an excess of points on the boundary, say $s^{3}$ such points. In this case, heuristically we expect

$$
\mathbb{E}\left(\left|\mathcal{C}\left(x ; x+Q_{s}\right)\right| \mid \mathcal{C}\left(x ; Q_{j}\right)\right) \approx s^{5} .
$$

Roughly, each point on the boundary gives rise to an expected $s^{2}$ points in $(x+$ $\left.Q_{s}\right) \backslash Q_{j}$, so assuming that the part of the cluster on the boundary is sufficiently spread out, they do not interfere negatively and you get (4.3). This of course means that $x$ is bad. We shall not justify these heuristics - they also require 
some additional assumptions — but we hope it gives the reader some intuition nonetheless. This example shows how our definition gives information about the behavior of the cluster $\mathcal{C}\left(x ; Q_{j}\right)$ on the boundary of $Q_{j}$. The alternative way, analyzing the behavior of the cluster on the boundary explicitly, while possible, is far more complicated.

We write $X_{j}^{K \text {-irr }}$ for

$$
X_{j}^{K \text {-irr }}=\mid\left\{x \in \partial Q_{j}: 0 \stackrel{Q_{j}}{\longleftrightarrow} x \text { and } x \text { is } K \text {-irregular }\right\} \mid .
$$

We are now ready to state the main theorem of this chapter.

Theorem 4. There exist constants $C>c>0$ such that for any $K$ sufficiently large and any $j$ and $M$ we have

$$
\mathbf{P}\left(X_{j} \geq M \text { and } X_{j}^{K-i r r} \geq X_{j} / 2\right) \leq C j^{d} \exp \left(-c \log ^{2} M\right) .
$$

Now is the time to skip to Chapter 5 .

4.2. Global and local regularity. Theorem 4 is the formulation needed in Chapter 5 to prove Theorem 2 It is natural to prove such a large deviation estimate using an exploration procedure which exploits the independence between different boxes in the lattice. However, the event defined in Definition 4.1 is a global definition, because we need to examine the edges of the entire cluster $\mathcal{C}\left(x ; Q_{j}\right)$ in order to determine it. To that aim, we define local events which can be determined by observing boxes of side length polynomial in $s$.

Definition 4.2. For $x \in \partial Q_{j}$ and a positive integer $s$, we say that the event $\mathcal{T}_{s}^{\text {loc }}(x)$ occurs if the following two happen:

(a) For all $y \in x+Q_{s}$,

$$
\left|\mathcal{C}\left(y ; x+Q_{s^{2 d}}\right) \cap\left(x+Q_{s}\right)\right|<s^{4} \log ^{4} s .
$$

(b) There exists at most $\log ^{3} s$ disjoint open paths starting in $x+Q_{s}$ and ending at $x+\partial Q_{s^{2 d}}$.

Note that in (a) we are interested in points in $x+Q_{s}$, but we allow the connecting paths to traverse in a much larger set, $x+Q_{s^{2 d}}$, but not unlimited. We immediately note

Claim 4.1. For any $x \in \mathbb{Z}^{d}$ and positive integer $s$,

$$
\mathcal{T}_{s}^{\text {loc }}(x) \Longrightarrow \mathcal{T}_{s}(x) \text {. }
$$

Proof. Indeed, assume to the contrary that

$$
\left|\mathcal{C}(x) \cap\left(x+Q_{s}\right)\right| \geq s^{4} \log ^{7} s,
$$

and let $X=\mathcal{C}(x) \cap\left(x+Q_{s}\right)$. We say that two vertices in $X$ are equivalent if there is an open path connecting them which does not exit $x+Q_{s^{2 d}}$. Due to (a) from the definition of $\mathcal{T}_{s}(x)$, each equivalence class contains at most $s^{4} \log ^{4} s$ vertices. Due to (b), there are no more than $\log ^{3} s$ equivalence classes, since each class requires its own path from $x+Q_{s}$ to the outside of $x+Q_{s^{2 d}}$ and all these paths are disjoint.

With this local version of $\mathcal{T}_{s}$, we are ready to give a local definition of badness. 

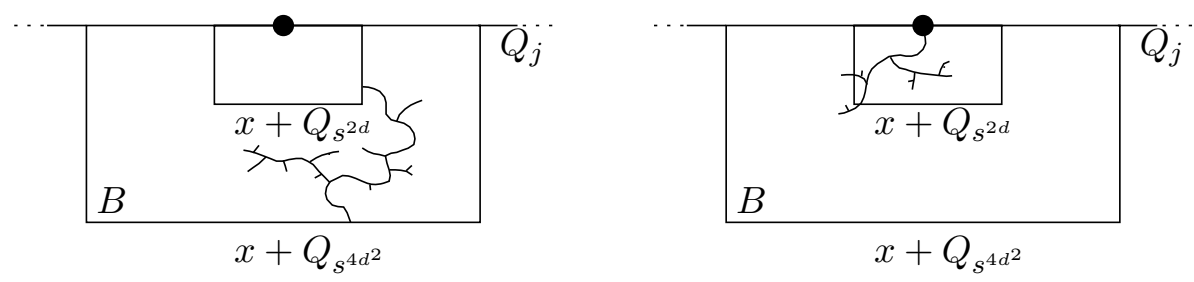

Figure 5. Spanning clusters. On the left, a cluster spanning from the outer boundary to the inner. On the right, the cluster containing $x$.

Definition 4.3. For $x \in \partial Q_{j}$ and positive integers $s$ and $K$ we define the following.

(i) We say that a cluster $\mathcal{C}$ in $B:=\left(x+Q_{s^{4} d^{2}}\right) \cap Q_{j}$ is a "spanning cluster" if $\mathcal{C} \cap Q_{j}$ intersects both $x+\partial Q_{s^{4 d^{2}}}$ and $x+\partial Q_{s^{2 d}}$. See Figure 5 . For notational convenience, we will also consider the cluster of $x$ as spanning even if it does not span anything.

(ii) We say that $x$ is $s$-locally-bad if there exist spanning clusters $\mathcal{C}_{1}, \ldots, \mathcal{C}_{m}$ in $B$ such that

$$
\mathbf{P}\left(\mathcal{T}_{s}^{\mathrm{loc}}(x) \mid \mathcal{C}_{1}, \ldots, \mathcal{C}_{m}\right) \leq 1-e^{-\log ^{2} s} .
$$

(iii) We say that $x \in \partial Q_{j}$ is $K$-locally-irregular if there exists $s \geq K$ such that $x$ is $s$-locally-bad. Otherwise we say that $x$ is $K$-locally-regular.

The importance of this definition is the fact that the event that $x$ is $s$-locally-bad is determined by the status of the edges in the box $\left(x+Q_{s^{4 d^{2}}}\right) \cap Q_{j}$. Let us proceed with observing that global goodness is implied by its local counterpart. We say that $x$ is $s$-good (s-locally-good) if it is not $s$-bad (s-locally-bad).

Claim 4.2. For any $x \in \partial Q_{j}$ and a positive integer $s$ we have that if $x$ is $s$-locallygood, then $x$ is $s$-good.

Proof. Assume to the contrary that $x$ is $s$-bad, i.e. that

$$
\mathbf{P}\left(\mathcal{T}_{s}(x) \mid \mathcal{C}\left(x ; Q_{j}\right)\right) \leq 1-\exp \left(-\log ^{2} s\right) .
$$

By Claim 4.1, $\mathcal{T}_{s}^{\text {loc }} \Longrightarrow \mathcal{T}_{s}$; therefore

$$
\mathbf{P}\left(\mathcal{T}_{s}^{\text {loc }}(x) \mid \mathcal{C}\left(x ; Q_{j}\right)\right) \leq 1-\exp \left(-\log ^{2} s\right) .
$$

Now, the event $\mathcal{T}_{s}^{\text {loc }}(x)$ depends only on what happens in $\mathcal{C}\left(x ; Q_{j}\right) \cap\left(x+Q_{s^{2 d}}\right)$, so

$$
\mathbf{P}\left(\mathcal{T}_{s}^{\text {loc }}(x) \mid \mathcal{C}\left(x ; Q_{j}\right)\right)=\mathbf{P}\left(\mathcal{T}_{s}^{\text {loc }}(x) \mid \mathcal{C}\left(x ; Q_{j}\right) \cap\left(x+Q_{s^{2 d}}\right)\right) .
$$

Examine now the cluster in the bigger box $x+Q_{s^{4 d^{2}}}$ and write it as a union of its components,

$$
\mathcal{C}\left(x ; Q_{j}\right) \cap\left(x+Q_{s^{4 d^{2}}}\right)=\mathcal{C}_{1} \cup \mathcal{C}_{2} \cup \ldots
$$

By definition, only the spanning clusters intersect the smaller box $x+Q_{s^{2 d}}$. Assume that the spanning clusters are $\mathcal{C}_{1}, \ldots, \mathcal{C}_{m}$. We get

so

$$
\mathcal{C}\left(x ; Q_{j}\right) \cap\left(x+Q_{s^{2 d}}\right)=\bigcup_{i=1}^{m} \mathcal{C}_{i} \cap\left(x+Q_{s^{2 d}}\right),
$$

$$
\mathbf{P}\left(\mathcal{T}_{s}^{\text {loc }}(x) \mid \mathcal{C}\left(x ; Q_{j}\right)\right)=\mathbf{P}\left(\mathcal{T}_{s}^{\text {loc }}(x) \mid \mathcal{C}_{1} \cap\left(x+Q_{s^{2 d}}\right), \ldots, \mathcal{C}_{m} \cap\left(x+Q_{s^{2 d}}\right)\right),
$$


and again by locality this equals

$$
\mathbf{P}\left(\mathcal{T}_{s}^{\text {loc }}(x) \mid \mathcal{C}_{1}, \ldots, \mathcal{C}_{m}\right) .
$$

Hence we get that $x$ is $s$-locally-bad (with these $\mathcal{C}_{1}, \ldots, \mathcal{C}_{m}$ ), in contradiction.

We write $X_{j}^{K \text {-loc-irr }}$ for

$$
X_{j}^{K \text {-loc-irr }}=\mid\left\{x \in \partial Q_{j}: 0 \stackrel{Q_{j}}{\longleftrightarrow} x \text { and } x \text { is } K \text {-locally-irregular }\right\} \mid .
$$

We will spend the rest of this chapter in proving the following theorem.

Theorem 5. There exists constants $C>c>0$ such that for any $K$ sufficiently large and any $j$ and $M$ we have

$$
\mathbf{P}\left(X_{j} \geq M \text { and } X_{j}^{K \text {-loc-irr }} \geq X_{j} / 2\right) \leq C j^{d} \exp \left(-c \log ^{2} M\right) .
$$

Proof of Theorem 4. This follows directly from Theorem 5 and Claim 4.2

4.3. An easy large deviation estimate. Our aim in this section is to prove the following lemma, which will be crucial for the proof of Theorem 5 .

Lemma 4.3. For $x \in \partial Q_{j}$ and positive integers $s$ we have

$$
\mathbf{P}(x \text { is s-locally-bad }) \leq C e^{-c \log ^{4} s} .
$$

In order to prove this lemma, we begin with a large deviation lemma.

Lemma 4.4. There exists some constant $c>0$ such that for all $s>0$ and $\lambda>0$ we have

$$
\mathbf{P}\left(\max _{y \in Q_{s}}\left|\mathcal{C}(y) \cap Q_{s}\right|>\lambda s^{4}\right) \leq s^{d-6} e^{-c \lambda} .
$$

Proof. Denote this maximum by $\mathcal{C}_{\max }$. Our lemma is a well-known corollary of the so-called "diagrammatic bounds". A convenient reference is [2, $\S 4.3$, lemma 2, eq. (4.12)]. It states that

$$
\mathbb{E}\left(\mathcal{C}_{\max }^{k}\right) \leq k ! C_{1}^{k} s^{d-6+4 k}
$$

(Aizenman's $\eta$ is simply 0 in our case). Using this with $k=\lambda /\left(2 C_{1}\right)$ gives

$$
\begin{aligned}
\mathbf{P}\left(\mathcal{C}_{\max }>\lambda s^{4}\right) & =\mathbf{P}\left(\mathcal{C}_{\max }^{k}>\left(\lambda s^{4}\right)^{k}\right) \leq \frac{\mathbb{E}\left(\mathcal{C}_{\max }^{k}\right)}{\left(\lambda s^{4}\right)^{k}} \leq k ! s^{d-6}\left(\frac{C_{1}}{\lambda}\right)^{k} \\
& \leq s^{d-6}\left(\frac{C_{1} k}{\lambda}\right)^{k}=s^{d-6} 2^{-\lambda / 2 C_{1}} .
\end{aligned}
$$

Proof of Lemma 4.3. Indeed, by Lemma 4.4, the probability of the complement of (a) from Definition 4.2 of $\mathcal{T}_{s}(x)$ is at most $C e^{-c \log ^{4} s}$. As for the complement of (b), by the volume estimate (1.1) we see that

$$
\begin{aligned}
\mathbf{P}\left(x+Q_{s}\right. & \left.\longleftrightarrow x+\partial Q_{s^{2 d}}\right) \\
& \leq \sum_{y \in x+\partial Q_{s}} \mathbf{P}\left(y \longleftrightarrow x+\partial Q_{s^{2 d}}\right) \\
& \leq \sum_{y \in x+\partial Q_{s}} \mathbf{P}\left(|\mathcal{C}(y)|>s^{2 d}-s\right)
\end{aligned}
$$




$$
\begin{aligned}
& \text { by (1.1) } \leq \sum_{y \in x+\partial Q_{s}} C\left(s^{2 d}-s\right)^{-1 / 2} \\
& \leq C s^{d-1} \cdot C s^{-d}=C / s .
\end{aligned}
$$

We now apply the BK inequality and we get that the probability of the complement of (b) is at most $(C / s)^{\log ^{3} s} \leq C e^{-c \log ^{4} s}$. We deduce that

$$
\mathbf{P}\left(\mathcal{T}_{s}(x)\right) \geq 1-C e^{-c \log ^{4} s} .
$$

Similarly to the above, for any $y \in x+\partial Q_{s^{2 d}}$, by (1.1) we have that

$$
\mathbf{P}\left(y \leftrightarrow x+\partial Q_{s^{4 d^{2}}}\right) \leq C s^{-2 d^{2}},
$$

whence the probability that there exists more than $\log ^{3} s$ spanning clusters (as in Definition 4.3) is at most

$$
\left(C s^{2 d(d-1)}\right)^{\log ^{3} s}\left(C s^{-2 d^{2}}\right)^{\log ^{3} s} \leq C e^{-c \log ^{4} s},
$$

since we have $C s^{2 d(d-1)}$ possible choices of $y$.

If $x$ is $s$-locally-bad, then either there are at least $\log ^{3} s$ spanning clusters, or there are at most $\log ^{3} s$ spanning clusters and a certain subset of them $\mathcal{C}_{1}, \ldots, \mathcal{C}_{m}$ (we assume here that these spanning clusters are numbered in some arbitrary fashion) has the property that

$$
\mathbf{P}\left(\mathcal{T}_{s}(x) \mid \mathcal{C}_{1}, \ldots, \mathcal{C}_{m}\right) \leq 1-e^{-\log ^{2} s} .
$$

However, for each such subset, by (4.5) we have that

$$
\mathbb{E} \mathbf{P}\left(\neg \mathcal{T}_{s}(x) \mid \mathcal{C}_{1}, \ldots, \mathcal{C}_{m}\right) \leq C e^{-c \log ^{4} s} .
$$

Thus, by the Markov inequality, the probability that $\mathcal{C}_{1}, \ldots, \mathcal{C}_{m}$ have the property (4.6) is at most $C \exp \left(\log ^{2} s-c \log ^{4} s\right) \leq C \exp \left(-c \log ^{4} s\right)$. We conclude the proof using the union bound, since $2^{\log ^{3} s} \cdot C e^{-c \log ^{4} s} \leq C e^{-c \log ^{4} s}$.

4.4. Exploring the cluster of the origin. In this subsection we prove Theorem 5. To that aim, we "explore" the cluster of the origin in $Q_{j}$ in boxes of size $s^{4 d^{2}}$; in fact we are only interested in the boundary $\partial Q_{j}$. This is quite a standard procedure, but let us describe it in detail. Let $w \in \mathbb{Z}^{d}$ be some shift, and let $G=G(w)$ be the collection of all cubes of size $2 s^{4 d^{2}}$, aligned to the shifted grid $w+\mathbb{Z}^{d}$ and intersecting $Q_{j}$, i.e.,

$$
G:=\left\{\left(Q_{2 s^{4 d^{2}}}+v\right) \cap Q_{j}: v \in\left(4 s^{4 d^{2}}+1\right) \mathbb{Z}^{d}+w\right\} \backslash\{\emptyset\},
$$

and choose an arbitrary ordering of $G$. The role of the shift $w$ is rather technical and will become evident later. The exploration process is a sequence of two subsets of $G, E_{i}$ (the explored boxes) and $A_{i}$ (the active boxes). We start with

$$
\begin{aligned}
& E_{1}=\left\{q \in G: q \cap \partial Q_{j}=\emptyset\right\}, \\
& A_{1}=\left\{q \in G: \exists x \in \partial q, 0 \stackrel{\cup E_{1}}{\longleftrightarrow} x\right\} \backslash E_{1},
\end{aligned}
$$

where we use the notation $\bigcup E_{i}=\bigcup_{q \in E_{i}} q$.

At step $i$ we choose from $A_{i-1}$ a box according to our ordering of $G$. Denote it by $q_{i}$. We add $q_{i}$ to the set of explored boxes $E_{i-1}$, and then add to $A_{i}$ all boxes 
not yet explored (that is, boxes not belonging to $E_{i-1}$ ) which can be reached from 0 by paths going only through the explored boxes $E_{i-1} \cup q_{i}$. Namely,

$$
\begin{aligned}
& E_{i}=E_{i-1} \cup\left\{q_{i}\right\}, \\
& A_{i}=\left(A_{i-1} \cup\left\{q \in G: \exists x \in \partial q, 0 \stackrel{\cup E_{i}}{\longleftrightarrow} x\right\}\right) \backslash E_{i} .
\end{aligned}
$$

Since the set of boxes $G$ is finite and $E_{i}$ increases at each step, at some time we must have $A_{i}=\emptyset$, at which time we cannot choose $q_{i+1}$. We say that the exploration finished, and we denote this stopping time by $\tau$.

The exploration process is used in order to define two martingales, one to control the number of bad boxes $\left(\beta_{i}\right)$ and one to control the boundary vertices $\left(\gamma_{i}\right)$. We say that a box $q \in G$ is $s$-bad if there exists some $x \in \partial Q_{j}$ which is $s$-locally-bad and such that $\left(x+Q_{s^{4 d^{2}}}\right) \cap Q_{j} \subset q$. Both martingales are adapted to the exploration filtration $\left\{\mathcal{F}_{i}\right\}$, namely to the configuration restricted to $\bigcup_{j<i} E_{j}$. Their definition is as follows. We start with $\beta_{1}=\gamma_{1}=0$. At each step we define

$$
\begin{aligned}
& \beta_{i}=\beta_{i-1}+\mathbf{1}\left\{q_{i} \text { is } s \text {-bad }\right\}-\mathbf{P}\left(q_{i} \text { is } s \text {-bad } \mid \mathcal{F}_{i-1}\right), \\
& \gamma_{i}=\gamma_{i-1}+\mathbf{1}\left\{\exists x \in q_{i} \cap \partial Q_{j}: 0 \stackrel{\cup E_{i}}{\longleftrightarrow} x\right\} \\
& \quad-\mathbf{P}\left(\exists x \in q_{i} \cap \partial Q_{j}: 0 \stackrel{\cup E_{i}}{\longleftrightarrow} x \mid \mathcal{F}_{i-1}\right) .
\end{aligned}
$$

We extend $\beta_{i}$ and $\gamma_{i}$ for all $i$ by making $\beta_{i}=\beta_{i-1}$ and $\gamma_{i}=\gamma_{i-1}$ when $A_{i-1}=\emptyset$. Clearly, they are indeed martingales. For our next lemmas, recall the definition of $X_{j}$ at (1.3).

Lemma 4.5. There exist constants $C_{1}>0$ and $c_{1}>0$ such that for any $j, s$ and $M$ we have

$$
\mathbf{P}\left(c_{1} e^{-C_{1} \log ^{2} s} \tau \geq X_{j} \geq M\right) \leq C e^{-c M+C \log ^{2} s},
$$

where $\tau$ is the stopping time for the exploration defined above.

When we apply the lemma, $s \ll M$, so you may think about the right-hand side as $C e^{-c M}$.

Proof. For every $i$,

$$
\begin{aligned}
X_{j} & \geq\left|\left\{k \leq i: \exists x \in q_{k} \cap \partial Q_{j}: 0 \stackrel{\cup E_{k}}{\longleftrightarrow} x\right\}\right| \\
& =\gamma_{i}+\sum_{k=1}^{i} \mathbf{P}\left(\exists x \in q_{k} \cap \partial Q_{j}: 0 \stackrel{\cup E_{k}}{\longleftrightarrow} x \mid \mathcal{F}_{k-1}\right) .
\end{aligned}
$$

To bound from below the sum on the right-hand side, note the fact that we explored $q_{k}$ implies that there exists some $z \in \partial q_{k}$ which is connected to 0 in $\bigcup E_{k-1}$. This means that given $\mathcal{F}_{k-1}$, the probability that there exists $x \in q_{k} \cap \partial Q_{j}$ such that $0 \stackrel{\cup E_{k}}{\longleftrightarrow} x$ is at least the probability that $z \stackrel{q_{k}}{\rightrightarrows} \partial Q_{j}$ which by Corollary 3.2 is at least $c e^{-C \log ^{2}\left(2 s^{4 d^{2}}\right)} \geq c_{2} e^{-C_{2} \log ^{2} s}$ for some $C_{2}>0$ and $c_{2}>0$. Assume also that $c_{2}<\frac{1}{2}$. This holds whenever the exploration at time $k$ is still alive. We will prove 
the assertion of the lemma with constants $c_{1}=c_{2}^{2}$ and $C_{1}=2 C_{2}$. For brevity write $\mu=c_{1} \exp \left(-C_{1} \log ^{2} s\right)$ and so we get that for any $i>0$,

$$
X_{j} \geq \gamma_{i}+\mu^{1 / 2} \min \{i, \tau\} .
$$

We rearrange to get that

$$
\begin{aligned}
\mathbf{P}\left(\mu \tau \geq X_{j} \geq M\right) & \leq \sum_{i=M / \mu}^{\infty} \mathbf{P}\left(\tau=i \text { and } X_{j} \leq \mu i\right) \\
& \leq \sum_{i=M / \mu}^{\infty} \mathbf{P}\left(\gamma_{i} \leq-\left(\mu^{1 / 2}-\mu\right) i\right) .
\end{aligned}
$$

We assumed $c_{2}<\frac{1}{2}$, which gives $\mu<\frac{1}{4}$ and hence $\mu^{1 / 2}-\mu \geq \mu^{1 / 2} / 2$. We now use the Azuma-Hoeffding inequality, which asserts that for any $a, \mathbf{P}\left(\gamma_{i} \leq-a\right) \leq e^{-a^{2} /(2 i)}$. We get that

$$
\mathbf{P}\left(\mu \tau \geq X_{j} \geq M\right) \leq \sum_{i=M / \mu}^{\infty} \exp (-\mu i / 8) \leq \frac{C}{\mu} e^{-c M},
$$

concluding the proof of the lemma.

The counterweight to Lemma 4.5 is the following lemma which estimates the bad vertices. Recall that $G$ and hence $\beta_{i}, \gamma_{i}$ and $\tau$ all depended on a parameter $w$, the shift. Now denote

$$
X_{j}^{s \text {-loc-bad }}=\mid\left\{x \in \partial Q_{j}: x \text { is s-locally-bad }\right\} \mid
$$

and

$$
X_{j}^{s-\operatorname{loc}-\text { bad }}(w)=\mid\left\{x \in \partial Q_{j}: x \text { is s-locally-bad, and } \exists q \in G(w) \text { s.t. } x \in q\right\} \mid .
$$

Of course, to conclude the proof of Theorem 5 we will need to use the fact that

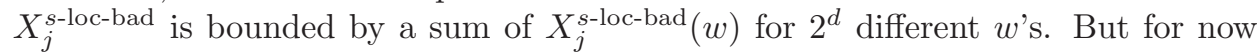
let us examine one $w$ only.

Lemma 4.6. There exist constants $C_{3}>0$ and $c_{3}>0$ such that for any $j, s, w$ and $M$, and for any real number $\mu \geq C_{3} e^{-c_{3} \log ^{4} s}$ we have

$$
\mathbf{P}\left(\mu^{-1} X_{j}^{s-l o c-b a d}(w) \geq \tau \geq M\right) \leq \frac{C}{s^{2-2 d} \mu^{2}} \exp \left(-c s^{2-2 d} \mu^{2} M\right) .
$$

Again, the way we apply this lemma, most of the factors on the right-hand side are negligible, and one can consider it as $\exp \left(-\mu^{2} M\right)$.

Proof. We have

$$
\beta_{i}=\mid\left\{k \leq i: q_{k} \text { is bad }\right\} \mid-\sum_{k=1}^{i} \mathbf{P}\left(q_{k} \text { is bad } \mid \mathcal{F}_{k-1}\right) .
$$

By Lemma 4.3 we have that

$$
\begin{aligned}
& \mathbf{P}\left(q_{k} \text { is bad } \mid \mathcal{F}_{k-1}\right) \\
& \leq \sum_{x: x+Q_{s^{4} d^{2}} \subset q_{k}} \mathbf{P}\left(x \text { is s-locally-bad } \mid \mathcal{F}_{k-1}\right) \\
& \text { by locality } \quad=\sum_{x: x+Q_{s^{4} d^{2}} \subset q_{k}} \mathbf{P}(x \text { is s-locally-bad })
\end{aligned}
$$




$$
\begin{aligned}
\text { by Lemma } 4.3 & \leq \sum_{x: x+Q_{s^{4} d^{2}} \subset q_{k}} C e^{-c \log ^{4} s} \\
& \leq C s^{4 d^{3}} \cdot C e^{-c \log ^{4} s} \leq C e^{-c \log ^{4} s}
\end{aligned}
$$

and so

$$
\beta_{i} \geq \mid\left\{k \leq i: q_{k} \text { is } \operatorname{bad}\right\} \mid-C e^{-c \log ^{4} s} i .
$$

Thus, it holds deterministically that

$$
X_{j}^{s-\text { loc-bad }}(w) \leq s^{d-1} \mid\left\{k \leq \tau: q_{k} \text { is bad }\right\} \mid \leq s^{d-1} \beta_{\tau}+C_{4} e^{-c_{4} \log ^{4} s} \tau .
$$

Define $c_{3}:=c_{4}$ and $C_{3}:=2 C_{4}$. We get that $X_{j}^{s-\operatorname{loc}-b a d}(w) \geq \mu \tau$ implies that

$$
\beta_{\tau} \geq s^{1-d} \tau\left(\mu-\frac{1}{2} C_{3} e^{-c_{3} \log ^{4} s}\right) \geq \frac{1}{2} s^{1-d} \mu \tau,
$$

by our assumption on $\mu$. This gives

$$
\begin{aligned}
\mathbf{P}\left(\mu^{-1} X_{j}^{s-l o c-b a d}(w)\right. & \geq \tau \geq M) \leq \mathbf{P}\left(\frac{2 s^{d-1}}{\mu} \beta_{\tau} \geq \tau \geq M\right) \\
& =\sum_{i=M}^{\infty} \mathbf{P}\left(\tau=i \text { and } \beta_{i} \geq \frac{1}{2} s^{1-d} \mu i\right) \\
& \leq \sum_{i=M}^{\infty} \mathbf{P}\left(\beta_{i} \geq \frac{1}{2} s^{1-d} \mu i\right) \\
& \leq \sum_{i=M}^{\infty} \exp \left(-c s^{2-2 d} \mu^{2} i\right) \leq \frac{C}{s^{2-2 d} \mu^{2}} \exp \left(-c \mu^{2} M s^{2-2 d}\right),
\end{aligned}
$$

where for the penultimate inequality we again used Azuma-Hoeffding.

Proof of Theorem [5, By definition,

$$
X_{j}^{K \text {-irr }} \leq \sum_{s \geq K}^{\infty} X_{j}^{s \text {-loc-bad }},
$$

so the theorem will be proved once we get a good estimate of

$$
\mathbf{P}\left(X_{j} \geq M \text { and } X_{j}^{s \text {-loc-bad }} \geq X_{j} / s^{2}\right)
$$

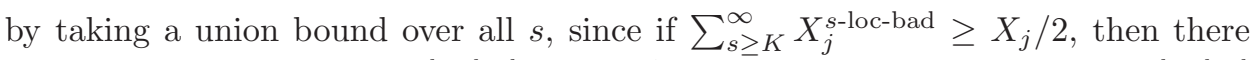
exists $s \geq K$ such that $X_{j}^{s \text {-loc-bad }} \geq X_{j} / s^{2}$ for $K \geq 3$. Next we relate $X_{j}^{s \text {-loc-bad }}$ to $X_{j}^{s \text {-loc-bad }}(w)$. Indeed, let $W=\left\{w: w_{i} \in\left\{0,2 s^{4 d^{2}}\right\} \forall i=1, \ldots, d\right\}$, so $W$ is a set of $2^{d}$ shifts. It is easy to convince oneself that for any $x \in \mathbb{Z}^{d}$ there exists some $w \in W$ such that $x+Q_{s^{4} d^{2}} \subset q$, where $q$ is a cube of the form $v+Q_{2 s^{4 d^{2}}}$, $v \in\left(4 s^{4 d^{2}}+1\right) \mathbb{Z}^{d}+w$. Thus

$$
X_{j}^{s-\text { loc-bad }} \leq \sum_{w \in W} X_{j}^{s-\operatorname{loc}-\text { bad }}(w),
$$

so it is enough to estimate $\mathbf{P}\left(X_{j} \geq M\right.$ and $\left.X_{j}^{s-\operatorname{loc}-\operatorname{bad}}(w)>X_{j} /\left(2^{d} s^{2}\right)\right)$. For this we write

$$
\begin{array}{r}
\mathbf{P}\left(X_{j} \geq M \text { and } X_{j}^{s \text {-loc-bad }}(w) \geq X_{j} /\left(2^{d} s^{2}\right)\right) \leq \mathbf{P}\left(c_{1} e^{-C_{1} \log ^{2} s} \tau \geq X_{j} \geq M\right) \\
+\mathbf{P}\left(2^{d} s^{2} X_{j}^{s-\operatorname{loc}-\operatorname{bad}}(w) \geq X_{j} \geq \max \left\{M, c_{1} e^{-C_{1} \log ^{2} s} \tau\right\}\right),
\end{array}
$$


where $c_{1}$ and $C_{1}$ are from Lemma 4.5. The first term on the right is estimated by Lemma 4.5 to be at most $C \exp \left(-c M+C \log ^{2} s\right)$. The event of the second term implies that

$$
X_{j}^{s-\text { loc-bad }}(w) \geq 2^{-d} s^{-2} c_{1} e^{-C_{1} \log ^{2} s} \tau
$$

and that $\tau \geq M s^{1-d}$, since $\tau \geq s^{1-d} X_{j}$. We wish to use Lemma 4.6 with $\mu=$ $2^{-d} s^{-2} c_{1} e^{-C_{1} \log ^{2} s}$ and $M_{\text {lemma } 4.6}=M s^{1-d}$. The only condition of Lemma 4.6 is that $\mu \geq C_{3} e^{-c_{3} \log ^{4} s}$, which will hold as long as $s$ is sufficiently large. We fix $K$ sufficiently large so that any $s \geq K$ will satisfy the requirement on $\mu$ and get by Lemma 4.6 that

$$
\begin{aligned}
\mathbf{P}\left(2^{d} s^{2} X_{j}^{s-\text { loc-bad }}(w)\right. & \left.\geq X_{j} \geq \max \left\{M, c_{1} e^{-2 C_{1} \log ^{2} s} \tau\right\}\right) \\
\leq & \frac{C}{\mu^{2} s^{2-2 d}} \exp \left(-c \mu^{2} M s^{3-3 d}\right) \leq C \exp \left(-c e^{-C \log ^{2} s} M+C \log ^{2} s\right) .
\end{aligned}
$$

This is the larger term, so we get

$$
\mathbf{P}\left(X_{j} \geq M \text { and } X_{j}^{s \text {-loc-bad }}(w)>X_{j} /\left(2^{d} s^{2}\right)\right) \leq C \exp \left(-c e^{-C \log ^{2} s} M+C \log ^{2} s\right) .
$$

We are nearly done. Let $s_{0}$ be the maximal $s$ such that $c e^{-C \log ^{2} s} \geq M^{-1 / 2}$, so $\log s_{0} \approx \log ^{1 / 2} M$. We have

$$
\begin{aligned}
\sum_{s=K}^{s_{0}} \mathbf{P}\left(X_{j}\right. & \left.\geq M \text { and } X_{j}^{s \text {-loc-bad }}(w)>X_{j} /\left(2^{d} s^{2}\right)\right) \\
& \leq \sum_{s=K}^{s_{0}} C \exp \left(-c e^{-C \log ^{2} s} M+C \log ^{2} s\right) \\
& \leq C \exp (-c \sqrt{M}+C \log M) \leq C e^{-c \sqrt{M}} .
\end{aligned}
$$

Summing over all $w \in W$ we get our estimate for small $s$,

$$
\sum_{s=K}^{s_{0}} \mathbf{P}\left(X_{j} \geq M \text { and } X_{j}^{s \text {-loc-bad }}>X_{j} / s^{2}\right) \leq C e^{-c \sqrt{M}} .
$$

For $s>s_{0}$ we use a much simpler estimate directly using Lemma 4.3 with no need to go through the "martingale lemmas" 4.5 and 4.6 and no need for $w$. We write

$$
\begin{aligned}
\mathbf{P}\left(X_{j}^{s \text {-loc-bad }}>M / s^{2}\right) & \leq \mathbf{P}\left(X_{j}^{s \text {-loc-bad }} \geq 1\right) \\
& \leq \sum_{x \in Q_{j}} \mathbf{P}(x \text { is } s \text {-loc-bad }) \leq C j^{d} \exp \left(-c \log ^{4} s\right),
\end{aligned}
$$

so

$$
\begin{aligned}
& \sum_{s=s_{0}}^{\infty} \mathbf{P}\left(X_{j} \geq M \text { and } X_{j}^{s \text { loc-bad }}>X_{j} / s^{2}\right) \\
& \leq \sum_{s=s_{0}}^{\infty} C j^{d} \exp \left(-c \log ^{4} s\right) \leq C j^{d} \exp \left(-c \log ^{2} M\right) .
\end{aligned}
$$

Clearly (4.8) is asymptotically larger than (4.7), so all-in-all we get

$$
\sum_{s=K}^{\infty} \mathbf{P}\left(X_{j} \geq M \text { and } X_{j}^{s \text {-loc-bad }}>X_{j} / s^{2}\right) \leq C j^{d} \exp \left(-c \log ^{2} M\right) .
$$


As already explained, this concludes the proof of Theorem [5. We write

$$
\begin{aligned}
\mathbf{P}\left(X_{j} \geq\right. & \left.M \text { and } X_{j}^{K \text {-loc-irr }} \geq X_{j} / 2\right) \\
& \leq \mathbf{P}\left(X_{j} \geq M \text { and }\left(\sum_{s \geq K} X_{j}^{s \text {-loc-bad }}\right) \geq X_{j} / 2\right) \\
& \leq \mathbf{P}\left(X_{j} \geq M \text { and } \bigcup_{s \geq K}\left\{X_{j}^{s \text {-loc-bad }} \geq X_{j} / s^{2}\right\}\right) \\
& \leq \sum_{s \geq K} \mathbf{P}\left(X_{j} \geq M \text { and }\left\{X_{j}^{s \text {-loc-bad }} \geq X_{j} / s^{2}\right\}\right) \\
& \leq C j^{d} \exp \left(-c \log ^{2} M\right)
\end{aligned}
$$

as required.

\section{Proof of Theorem 2}

Let $j$ and $L$ be as in Theorem 2 and let $K$ be some sufficiently large parameter; we will need it to be sufficiently large to allow us to apply Theorem 4 . but this is not the only restriction. We say that a pair of vertices $(x, y)$ are $(j, L, K)$-admissible if the following conditions hold (see Figure 6):

- $x \in \partial Q_{j}$ and $y \in x+Q_{L}$.

- $0 \stackrel{Q_{j}}{\longleftrightarrow} x$ and $x \leftrightarrow y$.

- $x$ is $K$-regular.

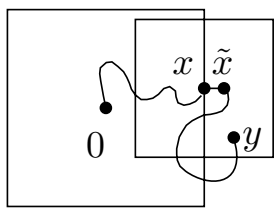

Figure 6. An admissible pair $(x, y)$.

- The edge $(x, \tilde{x})$ is pivotal for the event $0 \leftrightarrow y$, where $\tilde{x}$ is the neighbor of $x$ not in $Q_{j}$ (if more than one exists, choose the first in lexicographical order).

Define the random variable

$$
Y(j, L, K)=\mid\{(x, y):(x, y) \text { are }(j, L, K) \text {-admissible }\} \mid .
$$

We write $X_{j}^{K \text {-reg }}$ for the random variable counting the number of $K$-regular vertices, that is, $X_{j}^{K \text {-reg }}=X_{j}-X_{j}^{K \text {-irr }}$ (see the definition of $X_{j}^{K \text {-irr }}$ before the statement of Theorem (4). Throughout this section, $j, L$ and $K$ will be fixed, and we will usually omit them from the notation; namely, we will write $Y=Y(j, L, K), X^{\text {reg }}=X_{j}^{K \text {-reg }}$, etc. The following lemmas are the key steps in proving Theorem 2 .

Lemma 5.1. Let $K$ be sufficiently large, and let $j, M$ and $L$ be integers such that $M \geq L^{2} / 2$. Then there exists a constant $c=c(K)>0$ such that

$$
\mathbb{E} Y(j, L, K) \mathbf{1}_{\left\{X_{j}^{K-\text { reg }}=M\right\}} \geq c M L^{2} \mathbf{P}\left(X_{j}^{K \text {-reg }}=M\right) .
$$

Lemma 5.2. Let $j, K, M$ and $L$ be integers. Then

$$
\mathbb{E} Y^{2}(j, L, K) \mathbf{1}_{\left\{X_{j}^{K-\text { reg }}=M\right\}} \leq C M^{2} L^{4} \mathbf{P}\left(X_{j}^{K-\text { reg }}=M\right) .
$$

We begin with proving Theorem 2 given the lemmas. 
Proof of Theorem 2, Recall the definitions of $X_{j}$ and $A_{j}$ preceding the statement of Theorem 2 and denote $X=X_{j}, A=A_{j}$, etc. We begin with

$$
\begin{aligned}
\mathbf{P}\left(X \geq L^{2} \text { and } A \leq c L^{4}\right) & \leq \mathbf{P}\left(X \geq L^{2} \text { and } X^{\mathrm{irr}} \geq L^{2} / 2\right) \\
& +\sum_{M \geq L^{2} / 2} \mathbf{P}\left(X^{\mathrm{reg}}=M \text { and } A \leq c L^{4}\right) .
\end{aligned}
$$

We will bound the first term using Theorem 4, and each summand on the righthand side we bound using a second moment argument with Lemmas 5.1 and 5.2. We first note that

$$
A \geq Y .
$$

Indeed, for each pair $(x, y)$ counted in $Y$ we have that $0 \leftrightarrow y$ holds. Furthermore, we required that for each pair $(x, y)$ counted in $Y$ the edge $(x, \tilde{x})$ is pivotal for $0 \leftrightarrow y$. This shows that $x$ must be unique: if both $x_{1}$ and $x_{2}$ satisfy this, then by the "chain of sausages" picture [16, p. 91], one of them (say $x_{2}$ ) must be in the cluster connected to zero only by the pivotal edge $\left(x_{1}, \tilde{x}_{1}\right)$, which contradicts the requirement that $0 \stackrel{Q_{j}}{\longleftrightarrow} x_{2}$. This shows (5.2).

Recall the inequality (see [14])

$$
\mathbf{P}(V>a) \geq \frac{(\mathbb{E} V-a)^{2}}{\mathbb{E} V^{2}}
$$

valid for any random variable $V \geq 0$ and $a<\mathbb{E} V$. We use this for the variable $Y$ conditioned on $X^{\text {reg }}=M$ and for $a=c M L^{2}$. Lemmas 5.1 and 5.2 give that

$$
\mathbf{P}\left(Y>c M L^{2} \mid X^{\mathrm{reg}}=M\right)>c,
$$

and the fact that $M \geq L^{2} / 2$ gives that there exist positive constants $c_{1}, c_{2}$, depending on $K$, such that

$$
\mathbf{P}\left(Y \geq c_{1} L^{4} \mid X^{\mathrm{reg}}=M\right) \geq c_{2} .
$$

We use this and the fact that $A \geq Y$ (5.2) to derive that

$$
\mathbf{P}\left(X^{\mathrm{reg}}=M \text { and } A \leq c L^{4}\right) \leq\left(1-c_{2}\right) \mathbf{P}\left(X^{\mathrm{reg}}=M\right) .
$$

Putting this back into (5.1) and using Theorem 4 gives that

$$
\begin{aligned}
\mathbf{P}\left(X \geq L^{2} \text { and } A \leq c L^{4}\right) & \leq C j^{d} e^{-c \log ^{2} L}+\left(1-c_{2}\right) \mathbf{P}\left(X^{\mathrm{reg}} \geq L^{2} / 2\right) \\
& \leq C e^{-c \log ^{2} j}+\left(1-c_{2}\right) \mathbf{P}\left(0 \leftrightarrow \partial Q_{j}\right),
\end{aligned}
$$

where we used the fact that $L \geq j^{1 / 10}$. The first term is negligible (recall Lemma 2.2 and the fact that our theorem is only supposed to hold for $j$ sufficiently large), and this concludes our proof.

We proceed with the proofs of Lemmas 5.1 and 5.2. To this aim we define the following events. In these definitions we always have $x \in \partial Q_{j}$ and $y \in x+Q_{L}$ and $x^{\prime}$ in the box $\left(x+Q_{K}\right) \backslash Q_{j+K / 2} ;$ see Figure 7.

$$
\begin{aligned}
\mathcal{E}_{1}(x, M, K) & =\left\{0 \stackrel{Q_{j}}{\longleftrightarrow} x, x \text { is } K \text {-regular and } X_{j}^{\text {reg }}=M\right\}, \\
\mathcal{E}_{2}\left(x, x^{\prime}, y\right) & =\left\{x^{\prime} \leftrightarrow y \text { off } \mathcal{C}\left(x ; Q_{j}\right)\right\}, \\
\mathcal{E}_{3}\left(x, x^{\prime}\right) & =\left\{\mathcal{C}(x) \cap \mathcal{C}\left(x^{\prime}\right)=\emptyset\right\} .
\end{aligned}
$$

In the following we sometimes abbreviate $\mathcal{E}_{1}, \mathcal{E}_{2}$ and $\mathcal{E}_{3}$. 


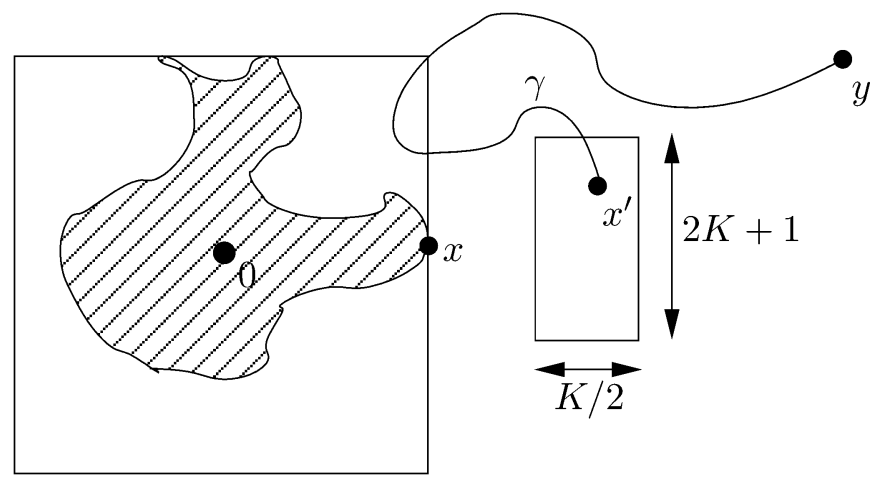

Figure 7 . The event $\mathcal{E}_{1}(x, M, K) \cap \mathcal{E}_{2}\left(x, x^{\prime}, y\right)$. The filled area is $\mathcal{C}\left(x ; Q_{j}\right)$; the box on the right is $\left(x+Q_{K}\right) \backslash Q_{j+K / 2}$.

Lemma 5.3. There exists a constant $c>0$ such that if $K>0$ is large enough, then for any $x \in \partial Q_{j}$ and any $x^{\prime} \in\left(x+Q_{K}\right) \backslash Q_{j+K / 2}$ we have that

$$
\sum_{y \in x+Q_{L}} \mathbf{P}\left(\mathcal{E}_{1} \cap \mathcal{E}_{2}\right) \geq c L^{2} \mathbf{P}\left(\mathcal{E}_{1}\right) .
$$

Proof. Note that $\mathcal{E}_{1}$ can be determined by observing only the edges of $\mathcal{C}\left(x ; Q_{j}\right)$. Hence we condition on $\mathcal{C}\left(x ; Q_{j}\right)=A$ and get that

$$
\mathbf{P}\left(\mathcal{E}_{1} \cap \mathcal{E}_{2}\right)=\sum_{A \text { admissible }} \mathbf{P}\left(\mathcal{C}\left(x ; Q_{j}\right)=A\right) \cdot \mathbf{P}\left(x^{\prime} \leftrightarrow y \text { off } A \mid \mathcal{C}\left(x ; Q_{j}\right)=A\right),
$$

where by $A$ admissible, we mean $A$ in which $\mathcal{E}_{1}$ holds and $\mathbf{P}\left(\mathcal{C}\left(x ; Q_{j}\right)=A\right)>0$. Since the event $\left\{x^{\prime} \leftrightarrow y\right.$ off $\left.A\right\}$ depends only on the status of edges not touching $A$ we have that

$$
\mathbf{P}\left(x^{\prime} \leftrightarrow y \text { off } A \mid \mathcal{C}\left(x ; Q_{j}\right)=A\right)=\mathbf{P}\left(x^{\prime} \leftrightarrow y \text { off } A\right) .
$$

Continuing we write

$$
\mathbf{P}\left(x^{\prime} \leftrightarrow y \text { off } A\right)=\mathbf{P}\left(x^{\prime} \leftrightarrow y\right)-\mathbf{P}\left(x^{\prime} \leftrightarrow y \text { only on } A\right) .
$$

If $x^{\prime} \leftrightarrow y$ only on $A$, then there exists $z \in A$ such that $\left\{x^{\prime} \leftrightarrow z\right\} \circ\{z \leftrightarrow y\}$. This together with the 2-point function estimate (1.2) gives that

$$
\mathbf{P}\left(x^{\prime} \leftrightarrow y \text { only on } A\right) \leq C \sum_{z \in A}\left|z-x^{\prime}\right|^{2-d}|z-y|^{2-d} .
$$

We sum this over $y$ and get that

$$
\sum_{y \in x+Q_{L}} \mathbf{P}\left(x^{\prime} \leftrightarrow y \text { only on } A\right) \leq C L^{2} \sum_{z \in A}\left|z-x^{\prime}\right|^{2-d} .
$$

We separate the sum dyadically over $z$ according to the scale of $z$ 's distance from $x^{\prime}$ as follows. For a given $t \geq 0$ let

$$
A_{t}=A \cap\left(x^{\prime}+\left(Q_{2^{t}} \backslash Q_{2^{t-1}}\right)\right) .
$$

With this notation we can write

$$
\sum_{z \in A}\left|z-x^{\prime}\right|^{2-d} \leq C \sum_{t=\lceil\log (K / 2)\rceil}^{\infty}\left|A_{t}\right| 2^{t(2-d)},
$$


where we began the sum on $t$ from $\lceil\log (K / 2)\rceil$ because if $z \in A$, then $z \in Q_{j}$ and hence $\left|z-x^{\prime}\right| \geq K / 2$ by our assumption on $x^{\prime}$. By the same assumption, note that for any $s$ such that $s \geq K / 2$ we have that

$$
x^{\prime}+Q_{s} \subset x+Q_{2 s} .
$$

We now claim that

$$
\left|A_{t}\right|<2^{4(t+1)}(t+1)^{7} \quad \forall t \text { such that } 2^{t} \geq K / 2 .
$$

Indeed, if $\left|A_{t}\right| \geq 2^{4(t+1)}(t+1)^{7}$, then directly from the definition of $\mathcal{T}$ we have that $\mathbf{P}\left(\mathcal{T}_{2^{t+1}}(x) \mid \mathcal{C}\left(x ; Q_{j}\right)=A\right)=0$. This is what we termed in the discussion after Definition 4.2 a "simple" bad configuration. However, $A$ is admissible, whence $x$ is $K$-regular, and we get a contradiction, hence (5.7). Thus,

$$
\sum_{z \in A}\left|z-x^{\prime}\right|^{2-d} \leq C \sum_{t=\lceil\log (K / 2)\rceil}^{\infty} t^{7} 2^{t(6-d)} \leq C K^{6-d} \log ^{7} K .
$$

We put this back into (5.5), and sum (5.4) over $y$ using (1.2). We get that

$$
\begin{aligned}
\sum_{y \in x+Q_{L}} \mathbf{P} & \left(x^{\prime} \leftrightarrow y \text { off } A\right) \\
& \geq c\left(\sum_{y \in x+Q_{L}}\left|x^{\prime}-y\right|^{2-d}\right)-C L^{2} K^{6-d} \log ^{7} K \\
& \geq L^{2}\left(c-C K^{6-d} \log ^{7} K\right),
\end{aligned}
$$

and so when $K$ is chosen large enough we have that

$$
\sum_{y \in x+Q_{L}} \mathbf{P}\left(x^{\prime} \leftrightarrow y \text { off } A\right) \geq c L^{2},
$$

and putting this back into (5.3) gives the assertion of the lemma.

Our next step is the following easy estimate.

Claim 5.4. Let $B \subset \mathbb{Z}^{d}$ be a set of vertices. Let $x^{\prime}$ be a uniform random vertex chosen from a finite set $A$. Then for any integer $s$ we have

$$
\mathbb{E}\left|\left(x^{\prime}+Q_{s}\right) \cap B\right| \leq \frac{\left|Q_{s}\right||B|}{|A|} .
$$

Proof. Indeed, for any $w \in Q_{s}$ we have that $\mathbf{P}\left(x^{\prime}+w \in B\right) \leq|B||A|^{-1}$.

Lemma 5.5. There exists a constant $c>0$ and $K>0$ large enough such that for any $x \in \partial Q_{j}$ there exists $x^{\prime} \in\left(x+Q_{K}\right) \backslash Q_{j+K / 2}$ with

$$
\sum_{y \in x+Q_{L}} \mathbf{P}\left(\mathcal{E}_{1} \cap \mathcal{E}_{2} \cap \mathcal{E}_{3}\right) \geq c L^{2} \mathbf{P}\left(\mathcal{E}_{1}\right) .
$$

Remark. The statement in fact holds for any $x^{\prime} \in\left(x+Q_{K}\right) \backslash Q_{j+K / 2}$ but proving this takes an extra effort. We only require one such $x^{\prime}$ and choosing $x^{\prime}$ at random simplifies the proof of this lemma significantly.

Proof. Let $x^{\prime}$ be a uniform random vertex in $(x+K)+Q_{K / 2}$ and $\mathbb{E}_{x^{\prime}}$ be the expectation operator with respect to this random choice. We will prove that

$$
\mathbb{E}_{x^{\prime}}\left[\sum_{y \in x+Q_{L}} \mathbf{P}\left(\mathcal{E}_{1} \cap \mathcal{E}_{2} \cap \mathcal{E}_{3}\right)\right] \geq c L^{2} \mathbf{P}\left(\mathcal{E}_{1}\right),
$$




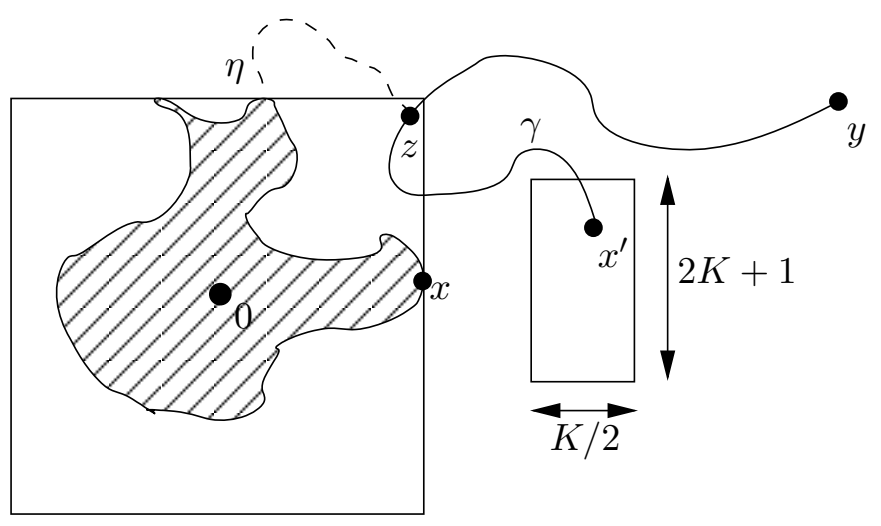

Figure 8 . The event $\mathcal{E}_{1} \cap \mathcal{E}_{2} \cap \neg \mathcal{E}_{3}$. The solid line between $x^{\prime}$ and $y$ is the path $\gamma$, and the dashed line is the path $\eta$.

and it follows that there exists $x^{\prime}$ such that the assertion of the lemma holds.

For any $x^{\prime}$, assume that $\mathcal{E}_{1} \cap \mathcal{E}_{2} \cap \neg \mathcal{E}_{3}$ occurs. We claim that in this case there exists a vertex $z$ such that the event

$$
\left\{\mathcal{E}_{1} \cap 0 \leftrightarrow z\right\} \circ\left\{x^{\prime} \leftrightarrow z\right\} \circ\{z \leftrightarrow y\}
$$

occurs. Indeed, let $\gamma$ be an open path between $x^{\prime}$ and $y$ which avoids $\mathcal{C}\left(x ; Q_{j}\right)$. Since we assume that $\neg \mathcal{E}_{3}$ occurs (that is, we assume $x \leftrightarrow x^{\prime}$ ) there must exist an open path $\eta$ connecting a vertex on $\mathcal{C}\left(0 ; Q_{j}\right)$ to a vertex on $\gamma$ such that, considered as sets of edges, $\eta \cap\left(\mathcal{C}\left(0 ; Q_{j}\right) \cup \gamma\right)=\emptyset$. Denote by $z$ the end vertex of $\eta(z$ is a vertex on the path $\gamma$ ). To verify (5.8) we check that the three events can be verified with a disjoint set of edges. Indeed, to verify $\mathcal{E}_{1} \cap\{0 \leftrightarrow z\}$ it suffices to observe the edges of $\mathcal{C}\left(x ; Q_{j}\right)$ and $\eta$. Note that "the edges of $\mathcal{C}\left(x ; Q_{j}\right)$ " means all edges needed to calculate $\mathcal{C}\left(x ; Q_{j}\right)$ precisely, i.e. all open edges inside the cluster and all closed edges defining its boundary in $Q_{j}$. To verify $\left\{x^{\prime} \leftrightarrow z\right\}$ we observe the edges of $\gamma$ up to $z$, and to verify $\{z \leftrightarrow y\}$ we observe the edges of $\gamma$ from $z$ to $y$. See Figure 8. The BK-Reimer inequality gives that

$$
\mathbf{P}\left(\mathcal{E}_{1} \cap \mathcal{E}_{2} \cap \neg \mathcal{E}_{3}\right) \leq \sum_{z} \mathbf{P}\left(\mathcal{E}_{1} \cap 0 \leftrightarrow z\right) \mathbf{P}\left(x^{\prime} \leftrightarrow z\right) \mathbf{P}(z \leftrightarrow y) .
$$

We sum over $y$ and use the 2-point function estimate (1.2) to get that

$$
\sum_{y \in x+Q_{L}} \mathbf{P}\left(\mathcal{E}_{1} \cap \mathcal{E}_{2} \cap \neg \mathcal{E}_{3}\right) \leq C L^{2} \sum_{z} \mathbf{P}\left(\mathcal{E}_{1} \cap 0 \leftrightarrow z\right)\left|z-x^{\prime}\right|^{2-d} .
$$

To sum over $z$, as in the previous lemma, we separate the sum over $z$ according to the scale of the distance of $z$ from $x^{\prime}$ and condition on $\mathcal{C}\left(x ; Q_{j}\right)$. Define

$$
B_{t}\left(x^{\prime}\right)=\mathcal{C}(0) \cap\left(x^{\prime}+\left(Q_{2^{t}} \backslash Q_{2^{t-1}}\right)\right) .
$$

We get that (5.9) is bounded above by

$$
C L^{2} \mathbf{P}\left(\mathcal{E}_{1}\right) \sum_{t \geq 1} \sum_{A \text { admissible }} \mathbb{E}\left(\left|B_{t}\left(x^{\prime}\right)\right| \cdot \mathbf{1}_{\mathcal{C}\left(x ; Q_{j}\right)=A}\right) 2^{t(2-d)},
$$

where again by $A$ admissible, we mean $A$ in which $\mathcal{E}_{1}$ holds and $\mathbf{P}\left(\mathcal{C}\left(x ; Q_{j}\right)=A\right)>$ 0 . It is at this point that we finally use the full power of our definition of bad 
vertices. Assume $K$ is a power of two, and put $t_{0}=\log (K / 2)$. We first sum (5.10) over $t>t_{0}$. For such a $t$, as in (5.6) we have that $\left|B_{t}\left(x^{\prime}\right)\right| \leq\left|\mathcal{C}(0) \cap\left(x+Q_{2^{t+1}}\right)\right|$ for all $x^{\prime}$ and we split the estimate according to whether $\mathcal{T}_{2^{t+1}}(x)$ occurs. If it does occur, then by definition of $\mathcal{T}_{2^{t+1}}(x)$ and the fact that $x \leftrightarrow 0$ we have that $\left|\mathcal{C}(0) \cap\left(x+Q_{2^{t+1}}\right)\right| \leq C 2^{4 t} t^{7}$, whence

$$
\mathbb{E}\left(\left|B_{t}\left(x^{\prime}\right)\right| \cdot \mathbf{1}_{\mathcal{T}_{2^{t+1}}(x)} \mid \mathcal{C}\left(x ; Q_{j}\right)=A\right) \leq C 2^{4 t} t^{7} .
$$

On the other hand, since $x$ is $K$-regular it is not $2^{t+1}$-bad for $t>t_{0}$, so by Definition 4.2 ,

$$
\begin{aligned}
\mathbb{E}\left(\left|B_{t}\left(x^{\prime}\right)\right| \mathbf{1}_{\neg \mathcal{T}_{2^{t+1}}(x)} \mid \mathcal{C}\left(x ; Q_{j}\right)=A\right) & \\
& \leq\left|Q_{2^{t}}\right| \cdot \mathbf{P}\left(\neg \mathcal{T}_{2^{t+1}}(x) \mid \mathcal{C}\left(x ; Q_{j}\right)=A\right) \leq C 2^{t d} e^{-t^{2}} .
\end{aligned}
$$

This is negligible with respect to (5.11) and we learn that

$$
\mathbb{E}\left(\left|B_{t}\left(x^{\prime}\right)\right| \mid \mathcal{C}\left(x ; Q_{j}\right)=A\right) \leq C 2^{4 t} t^{7},
$$

for $t>t_{0}$ and all $x^{\prime}$.

Next we sum over $t \leq t_{0}$ and here is where we use the fact that $x^{\prime}$ is randomized. We perform a split similar to before, but consider a box of size $2^{t_{0}+1}=K$ rather than $2^{t+1}$. Namely, if $\mathcal{T}_{K}$ occurs, then $\left|\mathcal{C}(0) \cap\left(x+Q_{K}\right)\right| \leq(2 K)^{4} \log ^{7}(2 K)$ and by Claim 5.4 we have that for any $t \leq t_{0}$,

$$
\mathbb{E}_{x^{\prime}}\left|\mathcal{C}(0) \cap\left(x^{\prime}+Q_{2^{t}}\right)\right| \leq \frac{C 2^{t d} K^{4} \log ^{7} K}{K^{d}},
$$

where $B$ from Claim 5.4 was taken to be $\mathcal{C}(0) \cap\left(x+Q_{K}\right)$. We deduce that

$$
\mathbb{E}_{x^{\prime}} \mathbb{E}\left(\left|B_{t}\left(x^{\prime}\right)\right| \mathbf{1}_{\mathcal{T}_{K}(x)} \mid \mathcal{C}\left(x ; Q_{j}\right)=A\right) \leq \frac{C 2^{t d} K^{4} \log ^{7} K}{K^{d}} .
$$

The case of $\neg \mathcal{T}$ is as before. Since $x$ is $K$-regular, for all $t \leq t_{0}$ we have that

$$
\mathbb{E}\left(\left|B_{t}\left(x^{\prime}\right)\right| \mathbf{1}_{\neg \mathcal{T}_{K}(x)} \mid \mathcal{C}\left(x ; Q_{j}\right)=A\right) \leq C 2^{t d} e^{-t_{0}^{2}},
$$

which is again negligible, and we deduce that for any $t \leq t_{0}$,

$$
\mathbb{E}_{x^{\prime}} \mathbb{E}\left(\left|B_{t}\left(x^{\prime}\right)\right| \mid \mathcal{C}\left(x ; Q_{j}\right)=A\right) \leq \frac{C 2^{t d} K^{4} \log ^{7} K}{K^{d}} .
$$

We put this together with (5.12) and get that for any admissible $A$,

$$
\begin{aligned}
& \mathbb{E}_{x^{\prime}} \sum_{t \geq 1} \mathbb{E}\left(\left|B_{t}\left(x^{\prime}\right)\right| \mid\right.\left.\mathcal{C}\left(x ; Q_{j}\right)=A\right) 2^{t(2-d)} \\
& \leq C K^{4-d} \log ^{7} K \sum_{t \leq t_{0}} 2^{2 t}+C \sum_{t>t_{0}} 2^{t(6-d)} t^{7} \leq C K^{6-d} \log ^{7} K
\end{aligned}
$$

(recall that $t_{0}=\log (K / 2)$ ). We put this into (5.10) and that into (5.9) and conclude that

$$
\mathbb{E}_{x^{\prime}} \sum_{y \in x+Q_{L}} \mathbf{P}\left(\mathcal{E}_{1} \cap \mathcal{E}_{2} \cap \neg \mathcal{E}_{3}\right) \leq C L^{2} \mathbf{P}\left(\mathcal{E}_{1}\right) K^{6-d} \log ^{7} K .
$$

We now apply Lemma 5.3 and choose $K$ large enough and we are done. 


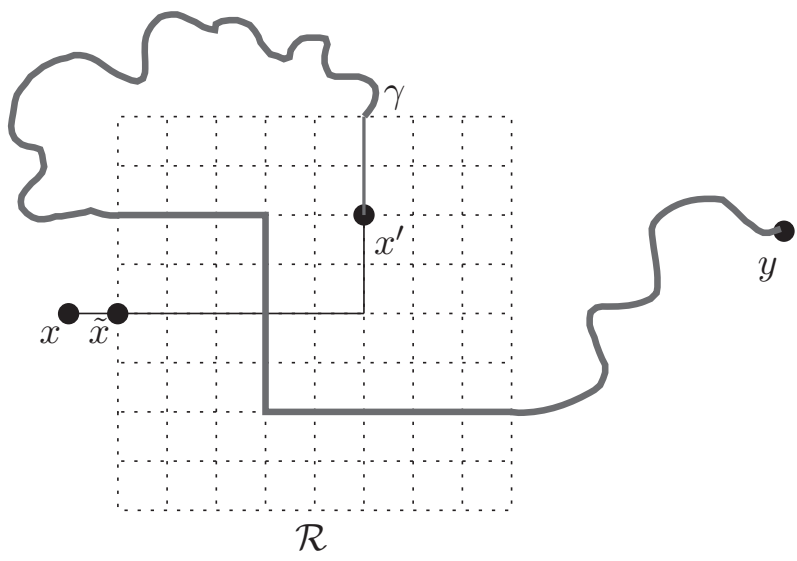

Figure 9. The local modification is performed in $\mathcal{R}$. The thick path connecting $x^{\prime}$ to $y$ is $\gamma$.

We are now ready to prove Lemmas 5.1 and 5.2 .

Proof of Lemma 5.1. The lemma will follow directly from Lemma 5.5 once we show that

$$
\begin{aligned}
& \mathbf{P}\left((x, y) \text { are }(j, L, K) \text {-admissible and } X_{j}^{\mathrm{reg}}=M\right) \\
& \quad \geq c(K) \mathbf{P}\left(\mathcal{E}_{1}(x, M, K) \cap \mathcal{E}_{2}\left(x, x^{\prime}, y\right) \cap \mathcal{E}_{3}\left(x, x^{\prime}\right)\right)
\end{aligned}
$$

for all $x$ and $y$ and $x^{\prime}$ chosen according to Lemma 5.5. Summing (5.13) over $y$ gives the $L^{2}$ factor, by Lemma 5.5 and the sum over $x$ obviously gives a factor of $M$. So we only need to show (5.13).

To show (5.13) we use a local modification argument as follows. Let $x, x^{\prime}$ and $y$ satisfy $\mathcal{E}_{1} \cap \mathcal{E}_{2} \cap \mathcal{E}_{3}$. Write $\gamma$ for the path connecting $x^{\prime}$ to $y$ which avoids $\mathcal{C}(x)$. Consider the edges in

$$
\mathcal{R}=\left(x+Q_{K}\right) \backslash Q_{j+1}
$$

so that $x^{\prime} \in \mathcal{R}$; see Figure 9. Let us now apply the following modification. Close all the edges in $\mathcal{R}$ except edges belonging to $\gamma$, and open the edges of an arbitrary path in $\mathcal{R}$ starting at $\tilde{x}$ (recall that $\tilde{x}$ is the neighbor of $x$ outside $Q_{j}$ ) and ending at $x^{\prime}$ (the thin path in Figure 9). Now open the edge $(x, \tilde{x})$. In the new configuration, $(x, y)$ is $(j, L, K)$-admissible and $X_{j}^{\text {reg }}$ is still equal to $M$. Indeed, $X_{j}^{\text {reg }}$ depends only on what happens inside $Q_{j}$ and we changed nothing there. For the same reason $x$ remains $K$-regular. The fact that in the original configuration $\mathcal{C}(x) \cap \mathcal{C}\left(x^{\prime}\right)=\emptyset$ ensures that the edge $(x, \tilde{x})$ is pivotal for $0 \leftrightarrow y$ in the modified configuration. Hence all conditions for admissibility are satisfied.

Now, in this modification, we changed the status of at most $(2 K)^{d}$ edges, which means that (5.13) holds with $c(K)=\left(\frac{1}{2} \min \left(p_{c}, 1-p_{c}\right)\right)^{(2 K)^{d}}$, and the lemma is proved. 
Proof of Lemma [5.2. If $\left(x_{1}, y_{1}\right)$ and $\left(x_{2}, y_{2}\right)$ are both $(j, K, L)$-admissible and $\left\{X_{j}^{\text {reg }}=M\right\}$ holds, then one of following three events occurs:

(i) $x_{1}=x_{2}, y_{1}=y_{2}$ and

$$
\left\{\mathcal{E}_{1}\left(x_{1}\right)\right\} \circ\left\{x_{1} \leftrightarrow y_{1}\right\},
$$

(ii) $x_{1}=x_{2}, y_{1} \neq y_{2}$ but both in $x+Q_{L}$ and there exists some $z$ such that

$$
\left\{\mathcal{E}_{1}\left(x_{1}\right)\right\} \circ\left\{x_{1} \leftrightarrow z\right\} \circ\left\{z \leftrightarrow y_{1}\right\} \circ\left\{z \leftrightarrow y_{2}\right\},
$$

(iii) $x_{1} \neq x_{2}, y_{1} \neq y_{2}, y_{i} \in x_{i}+Q_{L}$ and

$$
\left\{\mathcal{E}_{1}\left(x_{1}\right), \mathcal{E}_{1}\left(x_{2}\right)\right\} \circ\left\{x_{1} \leftrightarrow y_{1}\right\} \circ\left\{x_{2} \leftrightarrow y_{2}\right\} .
$$

To see this, first note that if $x_{1}=x_{2}$ and $y_{1} \neq y_{2}$, then one may consider the cluster $\mathcal{C}$ of all vertices connected to 0 only through $\left(x_{1}, \tilde{x}_{1}\right)$. By the definition of admissibility it contains both $y_{1}$ and $y_{2}$ and then one may define $z$ to be the triple point of $\tilde{x}_{1}, y_{1}$ and $y_{2}$ in $\mathcal{C}$ in the usual way. Since $\tilde{x}_{1} \notin Q_{j}$ we see that $\mathcal{C} \cap \mathcal{C}\left(0 ; Q_{j}\right)=\emptyset$ and hence the edges needed to define $\mathcal{C}\left(0 ; Q_{j}\right)$ - which are enough to prove that $\mathcal{E}_{1}\left(x_{1}\right)$ occurred - are disjoint from those defining the three paths between $x_{1}$ and $z, z$ and $y_{1}$, and $z$ and $y_{2}$. This shows (ii).

Assume now that $x_{1} \neq x_{2}$, and define $\mathcal{C}_{i}$ to be the cluster of vertices connected to 0 only through $x_{i}$. Both $\mathcal{C}_{1}$ and $\mathcal{C}_{2}$ are non-empty because $y_{i} \in \mathcal{C}_{i}$ and they are disjoint, because if $z \in \mathcal{C}_{1} \cap \mathcal{C}_{2}$, then taking a simple open path from $z$ to 0 and examining which of the edges $\left(x_{i}, \tilde{x}_{i}\right)$ it passes first, it is clear that it does not need to pass through the other, contradicting the definition of $\mathcal{C}_{i}$. Thus $\mathcal{C}_{1} \cap \mathcal{C}_{2}=\emptyset$ and we can choose open paths demonstrating that $x_{i} \leftrightarrow y_{i}$ which are both disjoint and disjoint from $\mathcal{C}\left(0 ; Q_{j}\right)$. This shows (iii) and the whole trichotomy.

We get that

$$
\mathbb{E} Y^{2} \mathbf{1}_{\left\{X^{\mathrm{reg}}=M\right\}} \leq S_{1}+S_{2}+S_{3},
$$

where

$$
\begin{aligned}
& S_{1}=\sum_{\substack{x \in \partial Q_{j} \\
y \in x+Q_{L}}} \mathbf{P}\left(\mathcal{E}_{1}(x)\right) \mathbf{P}(x \leftrightarrow y), \\
& S_{2}=\sum_{\substack{x \in \partial Q_{j} \\
y_{1}, y_{2} \in x+Q_{L}}} \mathbf{P}\left(\mathcal{E}_{1}(x)\right) \sum_{z} \mathbf{P}(x \leftrightarrow z) \mathbf{P}\left(z \leftrightarrow y_{1}\right) \mathbf{P}\left(z \leftrightarrow y_{2}\right), \\
& S_{3}=\sum_{\substack{x_{1}, x_{2} \in \partial Q_{j} \\
y_{i} \in x_{i}+Q_{L}}} \mathbf{P}\left(\mathcal{E}_{1}\left(x_{1}\right) \cap \mathcal{E}_{1}\left(x_{2}\right)\right) \mathbf{P}\left(x_{1} \leftrightarrow y_{1}\right) \mathbf{P}\left(x_{2} \leftrightarrow y_{2}\right) .
\end{aligned}
$$

Using (1.2) we easily estimate $S_{1}$ by

$$
S_{1} \leq C L^{2} \sum_{x \in \partial Q_{j}} \mathbf{P}\left(\mathcal{E}_{1}(x)\right)=C M L^{2} \mathbf{P}\left(X^{\mathrm{reg}}=M\right),
$$

where the last equality follows by definition of $\mathcal{E}_{1}$. To estimate $S_{2}$ we sum over $y_{1}$, $y_{2}$ and $z$ using Lemma 2.1 and get a term of $L^{6}$, so

$$
S_{2} \leq C M L^{6} \mathbf{P}\left(X^{\mathrm{reg}}=M\right) .
$$


Finally we use the 2-point estimate (1.2) to estimate $S_{3}$ and get

$$
\begin{aligned}
S_{3} & \leq \sum_{x_{1}, x_{2} \in \partial Q_{j}} \mathbf{P}\left(\mathcal{E}_{1}(x) \cap \mathcal{E}_{1}\left(x^{\prime}\right)\right) \sum_{y_{i} \in x_{i}+Q_{L}}\left|x_{1}-y_{1}\right|^{2-d}\left|x_{2}-y_{2}\right|^{2-d} \\
& \leq C L^{4} \sum_{x_{1}, x_{2} \in \partial Q_{j}} \mathbf{P}\left(\mathcal{E}_{1}(x) \cap \mathcal{E}_{2}\left(x^{\prime}\right)\right)=C M^{2} L^{4} \mathbf{P}\left(X^{\mathrm{reg}}=M\right) .
\end{aligned}
$$

We conclude that

$$
\mathbb{E} Y^{2} \mathbf{1}_{\left\{X^{\mathrm{reg}}=M\right\}} \leq C M^{2} L^{4} \mathbf{P}\left(X^{\mathrm{reg}}=M\right),
$$

since $M \geq L^{2} / 2$.

\section{Multiple ARMS}

The upper bound of $r^{-2 \ell}$ follows immediately from the BK inequality and so the main effort in this chapter is to prove the lower bound. To that aim we require an "inverse"-BK inequality. Our proof follows the standard proof of the BK inequality. Roughly, it starts with two identical copies of the graph, with one event on each copy, and then merging edges, and showing that the probability decreases with each merge. We will perform the same analysis on two copies of $Q_{2 r}$ but will only merge the edges of $Q_{r}$, and estimate how much is lost in each merge operation.

We begin by describing the setting, using the notation of [16]. Let $m>0$ be an integer and let $\Omega$ be the set of all $0-1$ vectors of length $m$. Let $\mathbf{P}$ be a product probability measure on $\Omega$ with density $p_{i}$ on the $i$-th coordinate, that is,

$$
\mathbf{P}(\omega)=\prod_{i=1}^{m}\left[\omega_{i} p_{i}+\left(1-\omega_{i}\right)\left(1-p_{i}\right)\right] \quad \forall \omega \in \Omega .
$$

Now, let $(\Omega, \mathbf{P})$ and $(\widetilde{\Omega}, \widetilde{\mathbf{P}})$ be two copies of $(\Omega, \mathbf{P})$ and write $\left(\Omega \times \widetilde{\Omega}, \mathbf{P}_{\otimes}\right)$ for the product space where $\mathbf{P}_{\otimes}=\mathbf{P} \times \widetilde{\mathbf{P}}$ is the product measure. Given two increasing events $\mathcal{A}$ and $\mathcal{B}$ in $\Omega$ write $\mathcal{A}^{\prime} \subset \Omega \times \widetilde{\Omega}$ for

$$
\mathcal{A}^{\prime}=\{(\omega, \widetilde{\omega}) \in \Omega \times \widetilde{\Omega}: \omega \in \mathcal{A}\},
$$

and $\mathcal{B}_{0}^{\prime} \subset \Omega \times \widetilde{\Omega}$ for

$$
\mathcal{B}_{0}^{\prime}=\{(\omega, \widetilde{\omega}) \in \Omega \times \widetilde{\Omega}: \widetilde{\omega} \in \mathcal{B}\} .
$$

For each $k \in\{1, \ldots, m\}$ write $\mathcal{B}_{k}^{\prime} \subset \Omega \times \widetilde{\Omega}$ for

$$
\mathcal{B}_{k}^{\prime}=\left\{(\omega, \widetilde{\omega}) \in \Omega \times \widetilde{\Omega}:\left(\omega_{1}, \ldots, \omega_{k}, \widetilde{\omega}_{k+1}, \ldots, \widetilde{\omega}_{m}\right) \in \mathcal{B}\right\} .
$$

In words, $\mathcal{B}_{k}^{\prime}$ is the event after merging the first $k$ edges. Note that $\mathbf{P}_{\otimes}\left(\mathcal{A}^{\prime} \circ\right.$ $\left.\mathcal{B}_{0}^{\prime}\right)=\mathbf{P}(\mathcal{A}) \mathbf{P}(\mathcal{B})$ and that $\mathbf{P}_{\otimes}\left(\mathcal{A}^{\prime} \circ \mathcal{B}_{m}^{\prime}\right)=\mathbf{P}(\mathcal{A} \circ \mathcal{B})$. The BK inequality follows immediately once one shows that for any $k$ we have $\mathbf{P}_{\otimes}\left(\mathcal{A}^{\prime} \circ \mathcal{B}_{k}^{\prime}\right) \leq \mathbf{P}_{\otimes}\left(\mathcal{A}^{\prime} \circ \mathcal{B}_{k-1}^{\prime}\right)$. The proof of this fact can be found in [16], but we do not need this here. What we will need is

$$
\mathbf{P}_{\otimes}\left(\mathcal{A}^{\prime} \circ \mathcal{B}_{k}^{\prime}\right)=\mathbf{P}_{\otimes}\left(\mathcal{A}^{\prime} \circ \mathcal{B}_{0}^{\prime}\right)-\sum_{i=1}^{k}\left[\mathbf{P}_{\otimes}\left(\mathcal{A}^{\prime} \circ \mathcal{B}_{i-1}^{\prime}\right)-\mathbf{P}_{\otimes}\left(\mathcal{A}^{\prime} \circ \mathcal{B}_{i}^{\prime}\right)\right] .
$$

In our setting, let $m$ be the number of edges which have at least one end in $Q_{2 r}$ and take $(\Omega, \mathbf{P})$ to be the usual Bernoulli percolation measure on these edges. Again, let $(\Omega, \mathbf{P})$ and $(\widetilde{\Omega}, \widetilde{\mathbf{P}})$ be two copies of $(\Omega, \mathbf{P})$ and $\left(\Omega \times \widetilde{\Omega}, \mathbf{P}_{\otimes}\right)$ to be the product measure. Let $e_{1}, \ldots, e_{m}$ and $\widetilde{e}_{1}, \ldots, \widetilde{e}_{m}$ be the edges corresponding to 
$\omega_{1}, \ldots, \omega_{m}$ and $\widetilde{\omega}_{1}, \ldots, \widetilde{\omega}_{m}$, respectively. We assume that the edges are ordered in such a way that there exists a number $k<m$ such that all the edges $e_{1}, \ldots, e_{k}$ and $\widetilde{e}_{1}, \ldots, \widetilde{e}_{k}$ have at least one end vertex in $Q_{r}$ and the rest of the edges have both endpoints not in $Q_{r}$.

Given $y_{1}, \ldots, y_{\ell} \in \mathbb{Z}^{d}$ as in the statement of Theorem 3 , with constant $K$ to be chosen later, we define the events $\mathcal{A}$ and $\mathcal{B}$ by

$$
\begin{aligned}
\mathcal{A} & =\left\{y_{1} \leftrightarrow \partial Q_{2 r}\right\} \circ \cdots \circ\left\{y_{\ell-1} \leftrightarrow \partial Q_{2 r}\right\} \\
\mathcal{B} & =\left\{y_{\ell} \leftrightarrow \partial Q_{2 r}\right\} .
\end{aligned}
$$

Lemma 6.1. Assume the setting of Theorem 3. Let $i \leq k$ and write $e_{i}=\left(z, z^{\prime}\right)$ for the corresponding edge in $Q_{r}$. Then

$$
\mathbf{P}\left(\mathcal{A}^{\prime} \circ \mathcal{B}_{i-1}^{\prime}\right)-\mathbf{P}\left(\mathcal{A}^{\prime} \circ \mathcal{B}_{i}^{\prime}\right) \leq C r^{-2 \ell} \sum_{j=1}^{\ell-1} \mathbf{P}\left(y_{j} \leftrightarrow z\right) \mathbf{P}\left(y_{\ell} \leftrightarrow z\right),
$$

where $C>0$ is a constant that depends on $\ell, d$ and the lattice chosen.

Proof. If $(\omega, \widetilde{\omega}) \in \mathcal{A}^{\prime} \circ \mathcal{B}_{i-1}^{\prime} \backslash \mathcal{A}^{\prime} \circ \mathcal{B}_{i}^{\prime}$, then we must have that $\widetilde{\omega}_{i}=1$. Consider the sets

$$
\mathcal{D}=\left\{(\omega, \widetilde{\omega}) \in \mathcal{A}^{\prime} \circ \mathcal{B}_{i-1}^{\prime} \backslash \mathcal{A}^{\prime} \circ \mathcal{B}_{i}^{\prime}: \widetilde{\omega}_{i}=1, \omega_{i}=0\right\}
$$

and

$$
\mathcal{D}^{\prime}=\left\{(\omega, \widetilde{\omega}) \in \mathcal{A}^{\prime} \circ \mathcal{B}_{i}^{\prime} \backslash \mathcal{A}^{\prime} \circ \mathcal{B}_{i-1}^{\prime}: \widetilde{\omega}_{i}=0, \omega_{i}=1\right\} .
$$

A moment's reflection shows that the map $\varphi$ which exchanges the values of $\omega_{i}$ and $\widetilde{\omega}_{i}$ is a one-to-one measure preserving map from $\mathcal{D}$ onto $\mathcal{D}^{\prime}$; indeed, both are characterized by the condition that any choice of the two sets $U$ and $V$ in the definition of o satisfies $\widetilde{e}_{i} \in V$ or $e_{i} \in V$, respectively. We deduce that

$$
\mathbf{P}\left(\mathcal{A}^{\prime} \circ \mathcal{B}_{i-1}^{\prime}\right)-\mathbf{P}\left(\mathcal{A}^{\prime} \circ \mathcal{B}_{i}^{\prime}\right) \leq \mathbf{P}\left(\left\{(\omega, \widetilde{\omega}) \in \mathcal{A}^{\prime} \circ \mathcal{B}_{i-1}^{\prime} \backslash \mathcal{A}^{\prime} \circ \mathcal{B}_{i}^{\prime}: \widetilde{\omega}_{i}=1, \omega_{i}=1\right\}\right) .
$$

If $\mathcal{A}^{\prime} \circ \mathcal{B}_{i-1}^{\prime} \backslash \mathcal{A}^{\prime} \circ \mathcal{B}_{i}^{\prime}$ occurs and $\widetilde{\omega}_{i}=\omega_{i}=1$, then $\mathcal{A}^{\prime}$ must use $e_{i}$ and $\mathcal{B}_{i-1}^{\prime}$ must use $\widetilde{e}_{i}$. This implies that for some $j \in\{1, \ldots, \ell-1\}$ we have that the events

- $\left\{y_{n} \leftrightarrow \partial Q_{2 r}\right\}$ for all $n \in\{1, \ldots, \ell-1\} \backslash\{j\}$ using the edges $e_{1}, \ldots, e_{m}$,

- $\left\{y_{j} \leftrightarrow z\right\} \cup\left\{y_{j} \leftrightarrow z^{\prime}\right\}$ using the edges $e_{1}, \ldots, e_{m}$

- $\left\{y_{\ell} \leftrightarrow z\right\} \cup\left\{y_{\ell} \leftrightarrow z^{\prime}\right\}$ using the edges $e_{1}, \ldots, e_{i-1}, \widetilde{e}_{i}, \widetilde{e}_{i+1}, \ldots, \widetilde{e}_{m}$

- $\left\{z \leftrightarrow \partial Q_{2 r}\right\} \cup\left\{z^{\prime} \leftrightarrow \partial Q_{2 r}\right\}$ using the edges $e_{1}, \ldots, e_{m}$,

- $\left\{z \leftrightarrow \partial Q_{2 r}\right\} \cup\left\{z^{\prime} \leftrightarrow \partial Q_{2 r}\right\}$ using the edges $e_{1}, \ldots, e_{i-1}, \widetilde{e}_{i}, \widetilde{e}_{i+1}, \ldots, \widetilde{e}_{m}$

occur disjointly. By the BK inequality we get that

$$
\begin{aligned}
& \mathbf{P}\left(\mathcal{A}^{\prime} \circ \mathcal{B}_{i-1}^{\prime} \backslash \mathcal{A}^{\prime} \circ \mathcal{B}_{i}^{\prime}\right) \\
& \quad \leq 16 \sum_{j \leq \ell-1}\left[\prod_{\substack{n \leq \ell-1 \\
n \neq j}} \mathbf{P}\left(y_{n} \leftrightarrow \partial Q_{2 r}\right)\right] \mathbf{P}\left(y_{j} \leftrightarrow z\right) \mathbf{P}\left(y_{\ell} \leftrightarrow z\right) \mathbf{P}\left(z \leftrightarrow \partial Q_{2 r}\right)^{2}
\end{aligned}
$$

(the factor 16 comes from the various possibilities to choose either $z$ or $z^{\prime}$ ). We now use Theorem 1 to conclude the proof of the lemma.

We are now ready to prove Theorem 3 .

Proof of Theorem 3 . We prove the claim by induction on $\ell$. The case $\ell=1$ is precisely Theorem 1. so we may assume $\ell \geq 2$. Recall the definition on $k$ and 
the events $\mathcal{A}$ and $\mathcal{B}$ from above. By Lemma 6.1 6.1] and the two-point function estimate (1.2) we get that

$$
\mathbf{P}_{\otimes}\left(\mathcal{A}^{\prime} \circ \mathcal{B}_{k}^{\prime}\right) \geq \mathbf{P}(\mathcal{A}) \mathbf{P}(\mathcal{B})-C r^{-2 \ell} \sum_{j=1}^{\ell-1} \sum_{z}\left|y_{j}-z\right|^{2-d}\left|y_{\ell}-z\right|^{2-d} .
$$

We sum this over $z$ and use the induction hypothesis to get that

$$
\mathbf{P}_{\otimes}\left(\mathcal{A}^{\prime} \circ \mathcal{B}_{k}^{\prime}\right) \geq c r^{-2 \ell}-C r^{-2 \ell} \ell K^{4-d} .
$$

Hence if we choose $K$ large enough (depending on $\ell$ ) we get that $\mathbf{P}_{\otimes}\left(\mathcal{A}^{\prime} \circ \mathcal{B}_{k}^{\prime}\right) \geq$ $c r^{-2 \ell}$. Now, since the edges $\left\{e_{i}\right\}_{i \leq k}$ are all the edges which have an endpoint in $Q_{r}$, the event $\mathcal{A}^{\prime} \circ \mathcal{B}_{k}^{\prime}$ implies that for all $j \leq \ell$ the events $y_{j} \leftrightarrow \partial Q_{r}$ occur disjointly using the edges $e_{1}, \ldots, e_{k}$. This concludes our proof.

\section{REFERENCES}

[1] Aharony, A. and Stauffer, D. (1991), Introduction To Percolation Theory, CRC Press.

[2] Aizenman, M. (1997), On the number of incipient spanning clusters. Nuclear Phys. B 485, no. 3, 551-582. MR1431856 (98j:82030)

[3] Aizenman, M. and Barsky, D. J. (1987), Sharpness of the phase transition in percolation models. Commun. Math. Phys. 108, no. 3, 489-526. MR874906 (88c:82026)

[4] Aizenman, M. and Newman, C. M. (1984), Tree graph inequalities and critical behavior in percolation models. J. Statist. Phys. 36, no. 1-2, 107-143. MR.762034 (86h:82045)

[5] Athreya, K. B. and Ney, P. E. (1927), Branching processes. Die Grundlehren der mathematischen Wissenschaften, Band 196. Springer-Verlag, New York-Heidelberg. MR 0373040 $(51: 9242)$

[6] Barsky, D. J. and Aizenman, M. (1991), Percolation critical exponents under the triangle condition. Ann. Probab. 19, no. 4, 1520-1536. MR1127713 (93b:60224)

[7] van den Berg, J. and Fiebig, U. (1987), On a combinatorial conjecture concerning disjoint occurrences of events. Ann. Probab. 15, no. 1, 354-374. MR877608(88d:60052)

[8] van den Berg, J. and Kesten, H. (1985), Inequalities with applications to percolation and reliability. J. Appl. Probab., 22, 556-569. MR799280 (87b:60027)

[9] Bollobs, B. and Riordan, O. (2006), Percolation. Cambridge University Press, New York. MR2283880(2008c:82037)

[10] Borgs, C., Chayes, J. T. and Randall, D. (1999), The van den Berg-Kesten-Reimer inequality: a review. Perplexing problems in probability, 159-173, Progr. Probab., 44, Birkhäuser Boston, Boston, MA, 1999. MR 1703130(2000j:60119)

[11] Brydges, D. and Spencer, T. (1985), Self-avoiding walk in 5 or more dimensions. Commun. Math. Phys., 97, no. 1-2, 125-148. MR 782962(86h:82056)

[12] Burton, R. M. and Keane, M. (1989), Density and uniqueness in percolation. Commun. Math. Phys., 121, no. 3, 501-505. MR990777 (90g:60090)

[13] Chayes, J. T. and Chayes, L. (1987), On the upper critical dimension of Bernoulli percolation. Commun. Math. Phys., 113, no. 1, 27-48. MR918403 (89c:82042)

[14] Durrett, R. (1996), Probability: Theory and Examples, Second edition. Duxbury Press, Belmont, California. MR1609153 (98m:60001)

[15] Erdős, P. and Rényi, A. (1960), On the evolution of random graphs, Magyar Tud. Akad. Mat. Kutató Int. Közl. 5, 17-61. MR0125031(23:A2338)

[16] Grimmett, G. (1999), Percolation. Second edition. Grundlehren der Mathematischen Wissenschaften, 321. Springer-Verlag, Berlin. MR1707339 (2001a:60114)

[17] Hara, T. (2008), Decay of Correlations in Nearest-Neighbour Self-Avoiding Walk, Percolation, Lattice Trees and Animals, Ann. Probab., 36, no. 2, 530-593. MR2393990 (2009c:82016)

[18] Hara, T., van der Hofstad, R. and Slade, G. (2003), Critical two-point functions and the lace expansion for spread-out high-dimensional percolation and related models, Ann. Probab., 31, no. 1, 349-408. MR.1959796 (2005c:60130)

[19] Hara, T. and Slade, G. (1990), Mean-field critical behaviour for percolation in high dimensions. Commun. Math. Phys., 128, no. 2, 333-391. MR1043524(91a:82037) 
[20] Hara, T. and Slade, G. (2000), The scaling limit of the incipient infinite cluster in highdimensional percolation. I. Critical exponents. J. Statist. Phys., 99, no. 5-6, 1075-1168. MR:1773141 (2001g:82053a)

[21] Harris, T. E. (1960), A lower bound for the critical probability in a certain percolation process. Proc. Cambridge Philos. Soc., 56, 13-20. MR0115221(22:6023)

[22] Heydenreich, M., van der Hofstad, R. and Sakai, A. (2008), Mean-field behavior for longand finite range Ising model, percolation and self-avoiding walk. J. Statist. Phys., 132, no. 6, 1001-1049. MR2430773(2009f:60116)

[23] van der Hofstad, R., den Hollander, F. and Slade, G. (2002), Construction of the incipient infinite cluster for spread-out oriented percolation above $4+1$ dimensions. Commun. Math. Phys., 231, 435-461. MR.1946445 (2003j:60141)

[24] van der Hofstad, R., den Hollander, F. and Slade, G. (2007), The survival probability for critical spread-out oriented percolation above $4+1$ dimensions. I. Induction. Probab. Theory Relat. Fields. 138, no. 3-4, 363-389. MR2299712 (2008g:60300)

[25] van der Hofstad, R., den Hollander, F. and Slade, G. (2007), The survival probability for critical spread-out oriented percolation above $4+1$ dimensions. II. Expansion. Ann. Inst. H. Poincaré Probab. Statist., 43, no. 5, 509-570. MR2347096 (2009d:60326)

[26] Kesten, H. (1980), The critical probability of bond percolation on the square lattice equals $\frac{1}{2}$. Commun. Math. Phys., 74, no. 1, 41-59. MR575895 (82c:60179)

[27] Kesten, H. (1982), Percolation theory for mathematicians. Progress in Probability and Statistics, 2. Birkhäuser, Boston, Mass. MR692943 (84i:60145)

[28] Kolmogorov, A. N. (1938), Zur Lösung einer biologischen Aufgabe [German: On the solution of a problem in biology]. Izv. NII Matem. Mekh. Tomskogo Univ. 2, 7-12.

[29] Kozma, G. and Nachmias, A. (2009), The Alexander-Orbach conjecture holds in high dimensions. Invent. Math., 178, no. 3, 635-654. MR 2551766

[30] Kozáková, Iva (2008), Critical percolation of free product of groups. Internat. J. Algebra Comput., 18, no. 4, 683-704. MR2428151(2009e:20057)

[31] Kozáková, Iva (2008 preprint), Critical percolation on Cayley graphs of groups acting on trees, http://arxiv.org/abs/0801.4153.

[32] Lawler, G., Schramm, O. and Werner, W. (2002), One-arm exponent for critical 2D percolation. Electron. J. Probab., 7, no. 2. MR 1887622(2002k:60204)

[33] Menshikov, M. V. (1986), Coincidence of critical points in percolation problems. (Russian) Dokl. Akad. Nauk SSSR, 288, no. 6, 1308-1311. English translation in: Soviet Math. Dokl. 33, no. 3, 856-859. MR 852458 (88k:60175)

[34] Nguyen, B. G. (1987), Gap exponents for percolation processes with triangle condition. J. Statist. Phys., 49, no. 1-2, 235-243. MR923855 (89e:60202)

[35] Reimer, D. (2000) Proof of the van den Berg-Kesten conjecture. Combin. Probab. Comput., 9, no. 1, 27-32. MR1751301 (2001g:60017)

[36] Sakai, A. (2004) Mean-field behavior for the survival probability and the percolation pointto-surface connectivity. J. Statist. Phys., 117, no. 1-2, 111-130. Erratum: J. Statist. Phys., 119 (2005), no. 1-2, 447-448. MR2098561 (2005h:60302) MR2144518

[37] Schonmann, R. H. (2001), Multiplicity of phase transitions and mean-field criticality on highly non-amenable graphs, Commun. Math. Phys., 219, no. 2, 271-322. MR 1833805 (2002h:82036)

[38] Slade, G. (2006), The lace expansion and its applications. Lectures from the 34th Summer School on Probability Theory held in Saint-Flour, July 6-24, 2004. MR2239599 (2007m:60301)

[39] Smirnov, S. (2001 preprint), Critical percolation in the plane. Available at http://www . unige . $\mathrm{ch} /{ }^{\sim}$ smirnov/papers/percol.ps.

[40] Smirnov, S. and Werner, W. (2001), Critical exponents for two-dimensional percolation. Math. Res. Lett., 8, no. 5-6, 729-744. MR1879816 (2003i:60173)

The Weizmann Institute of Science, Rehovot POB 76100, Israel

E-mail address: gady.kozma@weizmann.ac.il

Department of Mathematics, Massachusetts Institute of Technology, 77 MassachuSetts Avenue, Cambridge, Massachusetts 02139

E-mail address: asafnach@math.mit.edu 